\title{
Wir-Gefühle, Feindbilder und Feindseligkeit bei deutschsprachigen k.u.k. Soldaten im Ersten Weltkrieg. Eine emotionssoziologische Perspektive ${ }^{1}$
}

\author{
Sabine A. Haring
}

This paper deals with the We-feelings, the Images of Enemies, and the Hostility of German-speaking k.u.k. soldiers serving during the First World War. Against the background of Elias's figurational approach and in a sociology of emotions' perspective, my analysis is focused on affectual ties within military units and stresses the important role of different power balances (vertical loyalty) in regard to We-feelings and images of friends and enemies. In addition, group norms and group constraints - that influence wether the issue of We-feelings is broached or not - are rudimentary illustrated by using different sources: regimental histories, autobiographic manuscripts and diaries. These sources, as the paper shows, give a lively picture of a process from their initial enthusiasm to their growing alienation as a result of technicization and de-personalization and, finally, to their experiencing the war as either a natural, lawful necessity or, in contrast, as a pointless, meaningless struggle. The paper will particulary discuss the following questions: Which emotions are constitutive for We-Feelings and for Hostility at the front? What was the importance of emotions such as shame, sympathy, compassion and revenge regarding the bond of affection between soldiers of the Habsburg Army during World War I? Which role did „comradeship” play during attacks, particulary in regard to the escalation of violence at the front? And, how did the war shape the image of "the enemy"?

Figurational Sociology, Sociology of Emotions, We-Feelings, Images of Enemies, Hostility, First World War

Sabine A. Haring-Mosbacher is Associate Professor at the Department of Sociology at the Karl-Franzens-University of Graz, having received her $\mathrm{PhD}$ in social and economic sciences studies and completed her "habilitation" in sociology. Recent Publications: (with Helmut Kuzmics): Emotion, Habitus und Erster Weltkrieg. Soziologische Studien zum militärischen Untergang der Habsburger Monarchie (Göttingen 2013); Between the topos of a 'forgotten war' and the current memory boom: Remembering the First World War in Austria, in: Bart Ziino (Ed.): Remembering the First World War, S. 207-222 (London - New York 2015); "Nun natürlich begrüssten wir uns freundlichst und schlossen enge Kameradschaft". Wir-Gefühle im Ersten Weltkrieg unter emotionssoziologischer Perspektive. In: Gruppenpsychotherapie und Gruppendynamik, 51, 2015,

1 Eine detaillierte Darstellung und Analyse der folgenden Überlegungen findet sich bei Haring 2013, im Hinblick auf „Kameradschaft“ bei Haring 2014 und Haring 2015a. 
S. 174-190; (with Franz Höllinger, Anja Eder and Eva-Maria Griesbacher): Bäuerliche Lebenswelten in Österreich am Beginn des 21. Jahrhunderts (Graz 2017).

\section{Einleitende Bemerkungen}

Im Gedenkjahr 2014 waren die Beiträge zu Genese, Verlauf und Wirkungsgeschichte des von George Kennan als „Urkatastrophe des 20. Jahrhunderts“ bezeichneten Ersten Weltkriegs kaum mehr überblickbar. ${ }^{2}$ Zahlreiche wissenschaftliche und populärwissenschaftliche Neuerscheinungen sowie Neuauflagen bereits publizierter Werke erweiterten unser Wissen über den Ersten Weltkrieg ebenso wie die in alle großen - in unserem Fall - österreichischen Tageszeitungen ${ }^{3}$ erschienenen Reportagen zu Front und Hinterland zwischen 1914 und 1918 oder die in diversen Fernsehkanälen gezeigten Dokumentationen und historischen Filme ${ }^{4}$.

Seit dem Ende des Ersten Weltkriegs sind mittlerweile fast 100 Jahre vergangen, also etwa vier bis fünf Generationen. Die Rekonstruktion der Kriegserinnerungen von Soldaten, Offizieren und Zivilisten ist in jeder Generation - vor dem Hintergrund jeweils aktueller Zwangslagen und Opportunitäten - verschieden erfolgt und kann in ihrer Vollständigkeit nur als sozialer, intergenerationeller Prozess von Aufnahme und Weitergabe aufgefasst werden. Die kollektiven Erinnerungen an 1914-18 zu beachten, bedeutet also, sie als vier Generationen überspannenden Prozess zu rekonstruieren. ${ }^{5}$

Im Jahre 2014 gedachten die Staaten Europas dem Ausbruch des Ersten Weltkriegs vor 100 Jahren in ganz unterschiedlicher Art und Weise. Während in Großbritannien und Frankreich der Erste Weltkrieg in der Erinnerungskultur seinen festen Platz hat, zählt er in Österreich, wie Heidemarie Uhl unterstreicht,

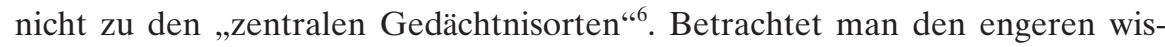

2 Jost Düffler gab einen guten Überblick über den „Historikerboom um den Ersten Weltkrieg“; vgl. Düffler 2014, S. 351-68. Ulrich Wyrwa bespricht in der Zeitschrift für Geschichtswissenschaft deutsche Neuerscheinungen zum Ersten Weltkrieg; vgl. Wyrwa 2016.

3 Vgl. u. a. „Schwerpunkt“, in: Der Standard, 30. November/1. Dezember 2013, und „1914 Der Erste Weltkrieg 1918. Spezial“, in: Kleine Zeitung, 10. Mai 2014.

4 Siehe u.a. „14 Tagebücher des Ersten Weltkriegs im Arte TV“ (vgl. http://www.14tagebuecher.de/page/de/about/1/ [download am 14. Mai 2014]), den ORF-Dokumentarfilm „Menschen \& Mächte: Der Weg in den Untergang“ (vgl. http://www.ots.at/pres seaussendung/OTS_20140423_OTS0103/100-jahre-erster-weltkrieg-menschen-maech te-dokumentiert-den-weg-in-den-untergang [download am 14. Mai 2014]) oder „Das Attentat Sarajevo 1914“, unter der Regie von Andreas Prochaska (vgl. https://presse portal.zdf.de/pm/das-attentat-sarajevo-1914 [download am 14. Mai 2014]).

5 Vgl. Haring / Kuzmics 2013, S. 15-16.

6 Vgl. Uhl 2013, S. 36. 
senschaftlichen Diskurs in Österreich, so lässt sich - wie Christa Hämmerle festhält - gegenwärtig beobachten, dass der

Erste Weltkrieg [...] von einer Randposition, die er im Vergleich zur dichten Erinnerungskultur des Zweiten Weltkriegs einnahm, wieder ins Zentrum des kollektiven Gedächtnisses an das ,Katastrophenzeitalter' des 20. Jahrhunderts rückt. ${ }^{7}$

Im Anbetracht dieser Fülle insbesondere in der jüngsten Vergangenheit erschienenen neuen Studien und der daraus gewonnenen neuen Erkenntnisse zum Ersten Weltkrieg stellt sich die Frage, was eine Soziologin noch Neues zur Weltkriegsforschung beizutragen vermag? Was meint sie, wenn sie „Wir-Gefühle, Feindbilder und Feindseligkeit bei deutschsprachigen k.u.k. Soldaten" unter einem emotionssoziologischen Fokus betrachten möchte? Und letztlich ist zu fragen, ob sich eine emotionssoziologische Betrachtungsweise lohnt und worin der „Mehrwert“ einer solchen Zugangsweise liegt?

\section{Krieg und Emotionen}

Der Weltkriegsboom rund um dieses Jubiläumsjahr darf uns zunächst nicht vergessen lassen, dass der Erste Weltkrieg in Zentraleuropa sehr lange ein ,vergessener Krieg“" war. Und noch immer gibt es in der Weltkriegsforschung zahlreiche Bereiche, die wenig untersucht sind: man denke in diesem Zusammenhang beispielsweise an Kindheiten im Ersten Weltkrieg. Betrachtet man die Fachdiskurse innerhalb der Soziologie, so zeigt sich zum einen, dass Krieg und Staatenkonkurrenz meist nur in spezialisierten Diskursen der Historischen Soziologie, der Militärsoziologie und der Soziologie des Krieges näher analysiert werden und deren Modelle und Befunde kaum Eingang in die Hauptströmungen der soziologischen Wissensproduktion finden. ${ }^{8}$ Zum anderen gibt es auch innerhalb der speziellen Soziologie „Emotionssoziologie“, deren Anfänge in Abgrenzung zur Psychologie vielfach mit den 1970er Jahren angesetzt werden und deren „Durchsetzungsphase“ Katharina Scherke um 1990 verortet, ${ }^{9}$ Themengebiete, die - mit wenigen Ausnahmen, man denke hier an Collins (2008) und Scheff (1994) - kaum Eingang in den emotionssoziologischen Diskurs gefunden haben. Dazu zählt unter anderem die Thematisierung von Erfahrungen in militärischen Verbänden sowohl im Krieg als auch im Frieden. ${ }^{10}$ In soziologischer Perspektive

\section{Hämmerle 2014, S. 9.}

8 Vgl. Haring / Kuzmics 2013, S. 44.

9 Vgl. Scherke 2009.

10 Dieser Befund ist insofern überraschend, als frühe, aus den 1970er Jahren stammende US-amerikanische emotionssoziologische Arbeiten, beeinflusst vom Interaktionismus und in Abgrenzung zum Parson'schen Funktionalismus, die Bedeutung und 
ist das Militär eine „Organisation zur kontrollierten und effizienten Anwendung von Gewalt“, wobei die „militärische Gehorsamsproduktion [...] in letzter Instanz darauf ausgerichtet [ist], diese Gewaltanwendung im Krieg, und hier vor allem in der Schlacht, zu gewährleisten "11. Der stark hierarchisch gegliederten Organisation liegen ,zwei unterschiedliche Strukturprinzipien zugrunde: das vertikale Prinzip von Befehl und Gehorsam und das horizontale der Kameradschaft ${ }^{\text {“12. }}$. Diese „Strukturprinzipien" fungieren als Werte bzw. moralische Codes, die von allen Armeeangehörigen sowohl im Krieg als auch im Frieden eingefordert werden. Im Rahmen der jeweiligen Ausbildung - vom Rekruten bis zum höheren Offizier - werden diese vermittelt und durch militärische Rituale verstärkt. Ziel ist deren Verinnerlichung, sodass „Befehlen“, „Gehorchen“ und „Kameradschaft bis in den Tod" in „Fleisch und Blut" übergehen und jeglicher Normverstoß zu Schuldgefühlen, Selbstzweifeln und schlechtem Gewissen des Individuums führt.

Trotz eines emotional turns haben wir es innerhalb der Emotionssoziologie also im Hinblick auf Erfahrungen im Militär mit einem Forschungsdesiderat zu tun. Sich mit dem Thema Emotionen von k.u.k. Soldaten im Ersten Weltkrieg zu beschäftigen, entspringt aber nicht nur dem Wunsch, das Augenmerk auf eine Forschungslücke zu legen, sondern ist in der Überzeugung begründet, dass Emotionen in der Wahrnehmung, in der Erfahrung, im sozialen Handeln und schließlich auch im Erinnern von Menschen, in unserem Fall von k.u.k. Soldaten im Ersten Weltkrieg, eine zentrale Rolle spielen. Denn Emotionen sind eine Orientierungshilfe für die Menschen. Sie tragen dazu bei, Menschen über ihre (fälschlich oft trennend-dualistisch konzipierte) Rationalität hinaus an die Welt zu binden. Emotionen reduzieren Handlungsalternativen durch Schaffen sozialer Nähe beziehungsweise Distanz, indem sie Objektbesetzungen nach dem dichotomen Schema angenehm/unangenehm, sympathisch/unsympathisch et cetera vornehmen, Grenzen zwischen ingroups und outgroups ziehen. Sie steuern soziales Verhalten (siehe affektuales Handeln). Im Prozess der Sozialisation erlernen Menschen nicht nur Normen, Werte, Rollen und Einstellungen, sondern auch emotionale Schemata. Geteilte Deutungsmuster legen zwar den Rahmen fest, was und wie in bestimmten Situationen gefühlt werden soll. Es existieren also so genannte feeling rules (Hochschild) ${ }^{13}$, die die Grenzen für das Fühlen selbst und für dessen Ausdruck abstecken. Der Prozess der Sinnstiftung vollzieht sich jedoch durch das Nadelöhr der beteiligten Akteure.

Funktion von Konflikten für gesellschaftliches Zusammenleben betonten. Man denke in diesem Zusammenhang u. a. an Cosers The Function of Social Conflict aus dem Jahre 1956. Siehe Senge 2013, S. 14; Haring 2011.

11 Meteling 2010, S. 26.

12 Ebd.

13 Vgl. Hochschild 1990. 
Das, was die Soziologin dabei in der Regel untersucht, sind Aussagen über Emotionen. Diese sind - wie Konstanze Senge unterstreicht - eine „Rekonstruktion höherer Ordnung“. ${ }^{14}$ Es geht also darum, wie Individuen Emotionen deuten, wobei die jeweiligen „Emotionskonzepte“ wiederum auf emotionale Erfahrung zurückwirken. Es geht mir aber nicht nur um Aussagen über Emotionen, sondern daneben gilt es auch die „Leerstellen“ in den Blick zu nehmen. Welche Gefühle werden selten oder gar nicht genannt, mit welchen Begriffen könnten diese aber umschrieben werden?

\subsection{Moral und Emotionen}

Sogenannte moralische Gefühle spielen im Krieg eine besondere Rolle. ${ }^{15}$ In der Einleitung ihres Aufsatzes „Moral Emotions“ kritisieren Jonathan H. Turner und Jan E. Stets enge Definitionen moralischer Gefühle:

The „moral emotions“ are often considered to be shame, guilt, sympathy, and empathy (Tangney and Dearing 2002), and, to a lesser degree, contempt, anger, and disgust (Rozin et al.1999), but a moment of reflection reveals that this view is far too narrow. The palate of human emotions is much larger and diverse than this short list of moral emotions; and since human capacities for emotion envolved to increase moral commitments to others, social structures and culture, many more emotions have moral effects. For example, righteousness, awe, veneration, joy, happiness, remorse, vengeance, and even sadness can mark emotional arousal of moral issues, as we hope to demonstrate. ${ }^{16}$

In einer soziologischen Perspektive versteht man unter Moral

ein mehr oder weniger umfassendes, integriertes System von Normen zur Beurteilung von individuellem oder sozialem Verhalten als ,richtig“ oder „falsch“, „gut“ oder „böse“, und zwar aufgrund spezifischer religiös-weltanschaulicher Orientierungen und soziokultureller Werte. ${ }^{17}$

Turner und Stets unterscheiden in diesem Zusammenhang je nach Reichweite und Intensität verschiedene Abstufungen „moralischer Codes“: „values“, „ideologies“, ,institutional norms“, „corporate unit norms“ and „situational norms“. Gerade an den Sanktionen, die beim Verstoß gegen die gültigen moralischen Codes folgen, kann man zunächst deren Existenz und an der Intensität der

14 Vgl. Senge 2013, S. 19.

15 Zum Verhältnis von moralischen Emotionen und moralischem Verhalten vgl. u.a. Tangney / Stuewig / Mashek 2007, die die Bedeutung von Emotionen für die „Übersetzung" moralischer Standards in moralisches Handeln unterstreichen.

16 Turner / Stets 2007, S. 544.

17 Hillmann 2007, S. 589. 
Sanktionen deren unterschiedliche Grade an Verbindlichkeit erkennen. Individuelle und kollektive Scham- und Schuldgefühle können dem Normverstoß folgen, die jedoch wiederum, wie Thomas Scheff eindrucksvoll zeigte, in Wut umschlagen können (Scham-Wut-Spirale). ${ }^{18}$ Das Befolgen und „Übererfüllen“ moralischer Codes wiederum macht das Individuum, die soziale Gruppe oder breite Teile der Gesellschaft stolz:

In effect, shame, guilt, embarrassment, and pride function as an emotional moral barometer, providing immediate and salient feedback on our social and moral acceptability. $[\ldots]$ When we „do the right thing", positive feelings of pride and self-approval are likely to result. ${ }^{19}$

Dabei sind Menschen in der Lage, ihre emotionalen Gefühlslagen vor dem Hintergrund ihrer Erfahrungen zu antizipieren. Diese Fähigkeit wiederum könne, so Tangney/Stuewig/Mashek, moralische Entscheidungen, also ob moralische Standards in moralische Handlungen übersetzt werden oder nicht, stark beeinflussen. ${ }^{20}$

Im Krieg tritt, wie Stietencron ausführt, für das Individuum eine Ausnahmesituation ein, indem das kulturelle Konstrukt, also der moralische Code des Tötungsverbotes durch das Tötungsgebot ersetzt wird. Während dem Individuum die Verfügung über menschliches Leben in der Regel nicht zusteht, kann die Gruppe ihre Zustimmung zum Töten im Krieg geben; die Tötungshemmung wird also, begleitet von unterschiedlichen Symboliken, aufgehoben. Die weitreichendste Form legitimierten Tötens von Menschen stellt der Krieg dar. Dabei nimmt derjenige, der tötet, jedoch auch das Selbst-Getötet-Werden in Kauf. ${ }^{21}$ Der von vielen konstatierten Tötungshemmung kann durch das gezielte Schaffen von Distanz beziehungsweise von Nähe begegnet werden: erstens durch die Schaffung von räumlicher Distanz, die zum Charakteristikum moderner Kriegsführung wurde und mit der Entwicklung von Distanzwaffen einhergeht; zweitens durch

18 Auf Scheff (1994) geht wiederum die Analyse einer Scham-Wut-Spirale zurück. Eine reale oder imaginierte Ablehnung könne eine Kettenreaktion von Scham und Wut auslösen; diese könne Sekunden, Stunden oder ein ganzes Leben andauern, in face to face Situationen, in Gruppen oder sogar zwischen Nationen auftreten. Wut könne dabei aggressiv nach außen oder nach innen im Sinne eines depressiven Rückzugs gerichtet sein. Sowohl zu wenig Distanz, also eine zu enge soziale Beziehung, als auch zu große Distanz können nach Scheff, gezeigt an Streitigkeiten zwischen Paaren sowie am Verhältnis zwischen Frankreich und Deutschland im Ersten und Zweiten Weltkrieg, als Bedrohung aufgefasst werden und Scham verursachen.

19 Tangney / Stuewig / Mashek 2007, S. 347. - Sighard Neckel bezeichnet Schuldgefühle und Scham als die beiden „psychischen Wachposten der Person“ (Neckel nach Scheve 2013, S. 237).

20 Vgl. ebd., S. 347.

21 Vgl. Stietencron 1995, S. 17-19. 
die Erzeugung sozialer Distanz durch die Herabsetzung des Gegners oder im Extremfall dadurch, dass ihm das Menschsein schlechthin abgesprochen wird (vgl. Feindbilder) ${ }^{22}$ und drittens durch den engen Gruppenzusammenhalt, durch die enge emotionale Bindung an die unmittelbare Gemeinschaft. ${ }^{23}$ Diese engen emotionalen Bindungen, diese Wir-Gefühle innerhalb militärischer Einheiten werden zumeist mit dem Begriff Kameradschaft beschrieben.

Im Hinblick auf die Ausübung kollektiver Gewalt muss der einzelne, wie Peter Imbusch herausarbeitet, durch die bereits vor Gewaltausübung veränderten Wertorientierungen keine persönliche Legitimierung entwickeln: „Stattdessen wird die Tat in spezifischer Weise als soziale Notwendigkeit, Pflichterfüllung, Befehlsnotstand oder über Gruppen- und Konformitätsdruck rationalisiert."Als weitere „Neutralisierungsmechanismen“ fungieren die Veränderung kollektiver moralischer Werte, die Dehumanisierung der Opfer, Desensibilisierungsprozesse in Bezug auf Gewaltausübung sowie die Entlastung von Verantwortlichkeit und die faktische Entkriminalisierung a priori. All diese Faktoren tragen zur Abschwächung, Relativierung oder gänzlichen Ausschaltung von Gewalt- und Tötungstabus bei, die bis zur „moralischen Anästhesie“ führen können. ${ }^{24}$

Im Krieg wird die Identifikation mit der eigenen Gruppe und ihrem „Wir-Bild“ (Norbert Elias) vorausgesetzt und immer wieder eingefordert und zwar in einer Intensität und Ausschließlichkeit, die sich von alltäglichen sozialen Interaktionen stark unterscheidet. Soldaten, aber nicht nur diese, sondern auch die Akteurinnen und Akteure an der Heimatfront, müssen ihre Bedürfnisse und Interessen den Zielen und Bedürfnissen des Kollektivs unterordnen. Innerhalb der unterschiedlichen militärischen Einheiten

wird die Rollenverteilung und -ausübung viel strenger und rigider vorgegeben und der Spielraum für individuelle Abweichungen ist sehr begrenzt. [...] Die wechselseitige Abhängigkeit wird zur existentiellen Grunderfahrung und alle unterliegen einem starken Zwang, sich mit den kollektiven Handlungs- und Denk-Normen zu identifizieren (Volkan 2013). Nicht nur der Körper soll für Ausnahmesituationen verfügbar sein, sondern

22 In diesem Zusammenhang kommt der Propaganda eine zentrale Rolle zu. Die von allen kriegsteilnehmenden Staaten stark eingesetzte Propaganda sollte zum einen Gruppenkohäsion und Selbstbild stärken und zum anderen den Feind in besonders negativem Licht erscheinen lassen. Kriegspropaganda versucht gerade mit den von ihr gezeichneten Feindbildern - beispielsweise die Russen als „Barbaren“, die Serben und Montenegriner als „schmutzige Hammeldiebe“ und „Schweinezüchter am Balkan“ (Lukan / Peyfuss 1983, S. 44) - bei den Empfängern bestimmte Emotionen hervorzurufen: Angst, Abscheu und Ekel sollen erzeugt werden, weniger bei den Männern, die in den Krieg ziehen wollen oder müssen, als vielmehr, wie Stietencron unterstreicht, bei den Frauen und Älteren, die ihre Männer und Söhne in den Krieg ziehen lassen sollen. Vgl. dazu insbesondere Jeismann 2009 und Stietencron 1995, S. 43-47.

23 Vgl. Herberg-Rothe 2003, S. 117-119.

24 Vgl. Imbusch 2002, S. 44. 
auch die Psyche und das ganze Leben. Jede Distanzierung bedeutet real und/oder symbolisch möglicherweise den Tod. ${ }^{25}$

\subsection{Wir-Gefühle, Feindbilder und Feinseligkeit}

„Wir-Gefühle, Feindbilder und Feinseligkeit“ im Krieg unter einem emotionssoziologischen Fokus zu analysieren, bedeutet danach zu fragen, welche Emotionen in der Beziehung zwischen Individuen, Gruppen oder Staaten, die in die „Gemeinschafts-“ und/oder „Freund-Kategorie“ sowie in die „Feind-Kategorie“ fallen, eine zentrale Rolle spielen. Es liegt die Vermutung nahe, dass man zwar auf der analytischen Ebene einzelne Emotionen wie Vertrauen, Bindungsgefühle, Stolz und Scham klar voneinander trennen kann, diese aber in der sozialen Wirklichkeit ineinander und miteinander verwoben sind. ${ }^{26}$ Dahinter steht die Annahme, dass sowohl „Freundschaft“ als auch „Feindschaft“ ganz bestimmte emotionale Bedürfnisse stillen, dass diese also nicht nur als etwas begriffen werden können, das für die handelnden Akteure ausschließlich durch Propaganda und Manipulation erst „erzeugt“ werden muss; oder um mit einem Klassiker der Soziologie, mit Georg Simmel, zu sprechen: Sowohl Freundschaft als auch Feindschaft sind Grundmuster der Vergesellschaftung. „Freund- und Feindbilder", so formuliert es der Pädagoge Max Liedtke,

haben den Menschen schon immer begleitet. Dabei spielte das „Feindbild“ die auffälligere Rolle. Es gehört zu den elementaren Erfahrungen des Menschen, dass nichts, was er hat und was ihm dieses Leben lebenswert erscheinen lässt, gesichert und unbedroht ist. Vielmehr erfährt er sich immer wieder als bedroht durch die Unbill der Natur, durch Krankheit und Tod, durch ein unerforschliches Schicksal, durch Tier und Mitmensch. Offenbar wegen dieser ständigen Erfahrung hat der Mensch sich die Welt stets nur in dualistischen Systemen zu erklären vermocht. [...] Das Freund-Schema spielt die gewichtigere, aber weniger auffällige Rolle. ${ }^{27}$

Nach Randall Collins ist gerade die emotionale Grenzziehung zwischen „Gemeinschaft" und „Nicht-Gemeinschaft" die bedeutsamste Dimension für Entscheidungen im Hinblick auf die Anwendung von Gewalt in sozialen Verhältnissen. Emotionale Besetzung der Gemeinschaft stiftet Solidarität nach innen und ermöglicht Feindschaft nach außen, wobei durch Rituale öffentlicher Gewaltdokumentation die emotionale Ausgrenzung sichtbar gemacht und die Integration nach innen gestärkt wird. Gefühle der Wärme, des Selbstvertrauens und des Enthusiasmus sind nach Collins die Solidargemeinschaft umschließende

25 Friedmann 2015, $195 \mathrm{f}$.

26 Einen umfassenden Überblick zu den einzelnen Emotionen im Hinblick auf WirGefühle, Feindbilder und Feinseligkeit gibt Haring 2013.

27 Vgl. Liedtke 2010, S. 19. 
Gefühle, die des Neides, des Hasses und der Wut nach außen gerichtete Gefühle. ${ }^{28}$ Emotionen sind also der „Lotse, der uns durch unbekanntes Terrain navigieren hilft. Sie sind das Zünglein an der Waage, wenn wir eine Entscheidung treffen. Sie erlauben uns blitzschnell zu sondieren, wem wir trauen können. Sie bilden den Klebstoff unserer sozialen Beziehungen. “29

\subsection{Das k.u.k. Militär als eine Vielvölkerarmee}

Eine Analyse der Emotionen von deutschsprachigen k.u.k. Soldaten im Ersten Weltkrieg muss die Charakteristika der k.u.k. Armee mitberücksichtigen. Das österreichisch-ungarische Heer war ein Spiegelbild der nationalen Verhältnisse in der Habsburger Monarchie. ${ }^{30}$ Von 1000 Soldaten waren 267 Deutsche, 223 Magyaren, 135 Tschechen, 85 Polen, 81 Ruthenen, 67 Kroaten und Serben, 64 Rumänen, 38 Slowaken, 26 Slowenen und 14 Italiener. ${ }^{31}$ Doch bedarf dieser Befund von Rothenberg noch einer differenzierteren Analyse. In den MilitärStatistischen Jahrbüchern von 1870 bis 1911 gibt es zwar eine Reihe von ,ethnischen Statistiken“, doch keine Angaben darüber, wie man zu den Statistiken kam. Bei den Rekruten - und damit bei den „Einjährig Freiwilligen“ - wurde bei der Einberufung die Muttersprache in die Statistiken eingetragen. Istvan Deák weist in diesem Kontext jedoch darauf hin, dass es zahlreiche zweisprachige Rekruten gegeben haben muss und hier die Vermutung nahe liegt, dass diese jene Sprache angaben, die die jeweiligen Behörden begrüßten. Die „einzige Kategorie“, in welcher es genaue Daten für den Zeitraum zwischen 1870 und 1911 gebe, sei die Kategorie der „einfachen Soldaten“ (für die „Mannschaften aktiv und in der

28 Vgl. Collins 2012, S. 121-156.

29 Wassmann 2002, S. 11.

30 Im Gegensatz zu den ,älteren Nationalstaaten“ wie England oder Frankreich oder den ,verspäteten Nationen“ wie Deutschland oder Spanien war die Habsburgermonarchie kein durch ethnische und sprachliche Homogenität gekennzeichneter Nationalstaat, sondern ein aus verschiedenen Ethnien zusammengesetzter Vielvölkerstaat. Ethnische Vielfalt, unterschiedliche staatlich-verfassungsmäßige Traditionen, unterschiedliche Religionszugehörigkeiten und Sprachen charakterisierten die Habsburgermonarchie. Viele Standardwerke über die letzten Jahre der Habsburgermonarchie heben die „unaufhaltsame" Sehnsucht der verschiedenen Nationalitäten auf Unabhängigkeit hervor, ein Wunsch, der letztendlich nicht im Rahmen der Struktur des Habsburgerreiches in Erfüllung gehen hätte können. Aber mit dieser Konzentration auf die zentrifugalen Kräfte, die die Monarchie auseinander brechen ließen, haben viele Forscher und Forscherinnen die zusammenhaltenden Kräfte, die die Monarchie am Leben hielten, einfach ignoriert. Eine detaillierte Beschreibung im Hinblick auf „zentrifugale“ und „zentripetale" Kräfte innerhalb der Habsburgermonarchie finden sich bei Haring 2013, S. 316-339. 31 Vgl. Rothenberg 1977, S. 74-75. 
Reserve“): ${ }^{32}$ Davon waren 25 Prozent Deutsche, 23,1 Prozent Magyaren, 16,5 Prozent Tschechen, Mährer und Slowaken, 7,9 Prozent Polen, 7,6 Prozent Ruthenen, 9 Prozent Kroaten und Serben, 2,4 Prozent Slowenen, 7 Prozent Rumänen, 1,3 Prozent Italiener und 385 (also weit unter einem Prozent) Bulgaren. Im Vergleich zum jeweiligen Bevölkerungsanteil in der Monarchie zeigt sich, dass Deutsche ( $+1,8$ Prozent), Magyaren ( $+3,5$ Prozent), Kroaten und Serben (+0,5 Prozent) und Rumänen $(+0,7 \%)$ überrepräsentiert, während Polen (-1,8 Prozent) unterrepräsentiert waren. Alle anderen Abweichungen zwischen dem jeweiligen Bevölkerungsanteil und dem Anteil innerhalb der „Mannschaften aktiv und in der Reserve" bewegten sich zwischen den Polen -0,5 Prozent und $+0,5$ Prozent. ${ }^{33}$ Bei den Reserveoffizieren hingegen waren - wie Deák zeigt Slowaken, Polen, Ruthenen, Kroaten, Serben und Rumänen deutlich unterrepräsentiert, während zumindest die Hälfte sich als „,deutsch“ deklarierte. Bei den Berufsoffizieren (vom Rang Leutnant bis Oberst) - folgt man den Militärstatistiken, deren Verlässlichkeit Deák stark bezweifelt - betrug der Anteil wesentlich mehr als 50 Prozent, nämlich 78,7 Prozent im Jahre 1910. Deák hingegen nimmt einen Anteil von Deutschen von circa 55 Prozent um 1900 an und geht davon aus, dass auch die anderen Nationalitäten, insbesondere die Magyaren, Tschechen sowie Serben und Kroaten ebenfalls unter den Berufsoffizieren stark vertreten waren. ${ }^{34}$ In der k.u.k. Armee hatten um 1900 Deutsch, Ungarisch, Tschechisch, Slowakisch, Polnisch, Ruthenisch, Serbo-kroatisch, Slowenisch, Rumänisch und Italienisch die „Anerkennung als Nationalsprachen“: Wenn mindestens 20 Prozent der Mannschaft eines Regiments eine bestimmte Sprache sprachen, mussten die Offiziere und Unteroffiziere ebenfalls diese Sprache „beherrschen“. Nur circa 80 grundlegende Befehle („Rechts“; „Links“; „Halt“ et cetera) wurden auf Deutsch gegeben. ${ }^{35}$ Die ,überwältigende Mehrheit der Einheiten der Habsburgerarmee“ waren - so Deák - vielsprachig. Im Hinblick auf die „Sprachenfrage“ in der k.u.k. Armee müsse man jedoch, wie Tamara Scheer jüngst betonte, drei Ebenen unterscheiden: die Ebene der Regimentssprache, die der Kommandosprache und die der Dienstsprache. ${ }^{36}$

Neben der Dynastie fungierte das nicht national, sondern dynastisch ausgerichtete Heer, wie Lawrence Cole ausführt, als einheitsstiftendes Band innerhalb der Monarchie, ja „für die pessimistischeren Konservativen am Hof, war am Vorabend des Ersten Weltkriegs die Armee sogar die letzte loyale Stellung im

32 Vgl. Deák 1991, S. 215-216.

33 Vgl. dazu das Militärgeschichtliche Jahrbuch für das Jahr 1910 (Wien 1911) nach Deák 1991, S. 216. - Zu den Unterschieden hinsichtlich der verschiedenen Waffengattungen vgl. ebd., S. 218.

34 Vgl. Deák 1991, S. 218-224.

35 Vgl. ebd., S. 122.

36 Vgl. Scheer 2014, S. 78-85. 
multinationalen Reich“37. Nach der Einführung der allgemeinen Wehrpflicht als „Schule des Patriotismus“ (Christa Hämmerle), „Schule der Männlichkeit“ (Christa Hämmerle) und „Schule der Emotion“ (Ute Frevert) schworen Millionen von Untertanen einen „Treueeid“ auf den Kaiser, den obersten Befehlshaber der Streitkräfte, der seine Macht nicht zuletzt mit Hilfe militärischer Symboliken und Rituale zur Schau stellte und sich persönlich zweifellos mit seiner Armee stark identifizierte. ${ }^{38}$ Als „Mediatoren ${ }^{\text {“639 }}$ von Treue und Loyalität entpuppten sich unter anderem auch Veteranenvereine, deren Gründungen gerade in den loyalitätsumstrittenen Grenzregionen (zum Beispiel dem Italienisch sprechenden Trentino) Möglichkeiten aber auch Grenzen der Vermittlung von Treue in der Peripherie zeigen.

Vor dem Hintergrund der zuvor kurz beschriebenen Charakteristika der Habsburgerarmee werden nun im Folgenden Wir-Gefühle, Feindbilder und Feinseligkeit deutschsprachiger k.u.k. Soldaten im Ersten Weltkrieg soziologisch untersucht. ${ }^{40}$ Die Figurationssoziologie und Zivilisationstheorie von Norbert Elias bilden dabei den theoretischen Hintergrund, der in dem von Helmut Kuzmics und mir 2013 veröffentlichten Buch Emotion, Habitus und Erster Weltkrieg ausführlich dargestellt wurde. ${ }^{41}$ Während dort die Analyse der Makroebene, die Ebene der Staaten und ihrer Konkurrenz breiten Raum einnimmt, liegt der Fokus hier auf der Mikroebene, der Gefühlswelt der Akteure an der Front: der deutschsprachigen k.u.k. Soldaten im Ersten Weltkrieg. Anhand von Regimentsgeschichten ${ }^{42}$ und Egodokumenten wird die komplexe Gemengelage im Hinblick auf „Wir-Gefühle, Feindbilder und Feindseligkeit“ auf Regiments-, Bataillons- und Kompanieebene beschrieben. Durch die Auswahl dieser unterschiedlichen Quellentypen - nämlich Regimentsgeschichten, autobiographischer

37 Vgl. Cole 2008, S. 350.

38 Vgl. Cole 2008, S. 350-353; Hämmerle 2012, S. 18 f.

39 Buschmann / Murr 2008, S. 33.

40 Ausführliche Analysen der „Entwicklungslinien und Typen des habsburgischen Offiziershabitus“ sowie des „k.u.k. Armeehabitus im Ersten Weltkrieg“, die unter anderem auf der Untersuchung zahlreicher Offiziersbiographien und autobiographischer Dokumente beruhen, finden sich bei Kuzmics 2013a und Kuzmics 2013 b.

41 Vgl. Kuzmics / Haring 2013.

42 Regimentsgeschichten wandten sich vorrangig an die Kriegskameraden (die durch ihren Kauf die Herausgabe überhaupt erst ermöglichten) und wohl auch an deren Familien. Seit den frühen 1920er Jahren erschien eine beachtliche Zahl an Regimentsgeschichten wie beispielsweise 1926 das „Ehrenbuch der Heßer: Geschichte des altösterreichischen Infanterie-Regimentes Freiherr von Heß Br. 49“ oder 1928 „Die Deutschmeister. Taten und Schicksale der Infanterieregiments Hoch- und Deutschmeister Nr. 4 insbesondere im Weltkriege“. In den 1930er Jahren lässt sich schließlich ein regelrechter Regimentsgeschichten-Boom beobachten; zahlreiche Regimentsgeschichten wurden verfasst, meist von ehemaligen k.u.k. Offizieren. Siehe Haring 2015a. 
Manuskripte ${ }^{43}$ und Tagebücher - sollen im Sinne einer Quellentriangulation die verschiedenen Perspektiven, die sich nicht zuletzt auch in den jeweiligen Quellengattungen manifestieren, stärker betont werden. ${ }^{44}$

\section{Wir-Gefühle im Krieg}

Am Beginn des 21. Jahrhunderts dominiert die Vorstellung, dass Soldaten für nationale Ziele oder zumindest aufgrund einer patriotischen Gesinnung kämpfen. Aber im Kampf sind nationale oder patriotische Ziele zu abstrakt, Soldaten kämpfen - wie unter anderen die Studie The American Soldier ${ }^{45}$ von Stouffer et. al. zeigte -, um die „Aufgabe zu beenden“ (ending the task) sowie aus Solidarität mit der Gruppe: $:^{46}$ „Es ist häufig vermutet worden“, so Niall Ferguson in seinem Buch Krieg der Welt, ,dies sei der eigentliche Schlüssel zum militärischen Zusammenhalt: nicht Patriotismus und noch nicht einmal die Treue zum Regiment, sondern ,Kameradschaft' - Treue zu seinen Gefährten in der kleinsten Kampfeinheit. “" ${ }^{47}$ Oder wie es Erich Maria Remarque in seinem weltberühmten Roman Im Westen nichts Neues seinen Erzähler Paul Bäumer formulieren lässt:

Das Wichtigste aber war, daß in uns [bereits in der Ausbildungszeit; Anm. d. Verf.] ein festes praktisches Zusammengehörigkeitsgefühl erwachte, das sich im Felde dann zum Besten steigerte, was der Krieg hervorbrachte: zur Kameradschaft ! ${ }^{48}$

Das eigentlich Erstaunliche an der Geschichte der Armeen im Ersten Weltkrieg sei, wie Wencke Meteling unterstreicht, das enorme Durchhaltevermögen der Armeen gewesen, das sich sowohl auf den Zwang zur Disziplin und das hohe

43 Zahlreiche autobiographische Manuskripte wurden vom „Dokumentationsarchiv lebensgeschichtlicher Aufzeichnungen, Institut für Wirtschafts- und Sozialgeschichte, Universität Wien“, gesammelt, unter der Ruprik „Bestand D: Kriegsbezogene Aufzeichnungen aWK1" zusammengefasst und Wissenschaftlerinnen und Wissenschaftlern dankenswerter Weise zu Forschungszwecken zur Verfügung gestellt. Vgl. http://wirt schaftsgeschichte.univie.ac.at/vereine/doku/dokumentenbestand/bestand-d/ [download am 7. September 2016]).

44 Sämtliche Quellenangaben finden sich in Kuzmics / Haring 2013, S. 565-569.

45 Stouffer et al. 1949.

46 Die befragten US-Soldaten sollten die für sie bedeutsamsten Motive zum Weitermachen angeben: „Generally, from your combat experience, what was most important to you in making you want to keep going and do as well as you could?“ „Die Aufgabe beenden“ nannten $39 \%$ der Gemeinen und $14 \%$ der Offiziere im Hinblick auf die Soldaten. „Solidarität mit der Gruppe“ gaben $14 \%$ der Soldaten und $15 \%$ der Offiziere wiederum in Bezug auf die Motive der Soldaten an. Vgl. Stouffer et al. 1949, S. 109.

47 Ferguson 2006, S. 194.

48 Remarque 1996, S. 29. 
Risiko bei Fahnenflucht als auch auf Kohäsionsfaktoren - esprit de corps, primary group cohesion, Kameradschaft - zurückführen lässt: „Kameradschaft [war] der Inbegriff zwischenmenschlicher Kohäsion im Militär." ${ }^{49}$ Während sich vor dem Ersten Weltkrieg innerhalb der Kompanien die Offiziere, Unteroffiziere und Soldaten persönlich näher kannten und sich mit ihren jeweiligen Einheiten oftmals stark identifizierten, ${ }^{50}$ wurde dieser „Korpsgeist im Verlauf des Krieges“ durch personelle Fluktuation als Folge von Verletzungen, Tod, Versetzungen und Abkommandierungen sowie Änderung der Rekrutierungspraxis - weg von der tendenziell lokalen Rekrutierung - zunehmend ausgehöhlt.

Am Beginn des 21. Jahrhunderts ist für die meisten Europäerinnen und Europäer jedoch „Kameradschaft“ „,ein Begriff wie aus einer anderen Welt“"51, auch wenn in Dienstvorschriften und Gesetzen von Heeren - wie beispielsweise in der Allgemeinen Dienstvorschrift für das Bundesheer (ADV), § 3 unter der Rubrik „Allgemeine Pflichten des Soldaten“ - „Kameradschaft“ eingefordert wird:

Der Soldat steht auf Grund der ihm übertragenen Aufgabe, sein Vaterland und sein Volk zu schützen und mit der Waffe zu verteidigen, in einem besonderen Treueverhältnis zur Republik Österreich. Er ist im Rahmen dieses Treueverhältnisses insbesondere zur Verteidigung der Demokratie und der demokratischen Einrichtungen sowie zu Disziplin, Kameradschaft, Gehorsam, Wachsamkeit, Tapferkeit und Verschwiegenheit verpflichtet. [...] Alle Soldaten haben ihren Kameraden mit Achtung zu begegnen, sie vor unnötiger Gefährdung zu bewahren und ihnen in Not und Gefahr beizustehen. ${ }^{52}$

Auch bei der Analyse soziologischer Lexika und Handwörterbücher zeigt sich, dass ältere Ausgaben - wie das 1969 von Bernsdorf in zweiter Auflage herausgegebene Wörterbuch der Soziologie - noch das Stichwort „Kameradschaft“" enthalten, ${ }^{53}$ jüngere Ausgaben jedoch keinen Eintrag mehr zu „Kameradschaft“" aufweisen. So unterscheidet Bernsdorf 1969 im Artikel „Kameradschaft“54 zwi-

49 Meteling 2010, S. 275.

50 So meldeten sich, wie Meteling in ihrer Untersuchung über deutsche und französische Regimenter zeigt, 1914 viele Freiwillige zu ganz bestimmten Regimentern. Heinz von Lichem betont in seiner Regimentsgeschichte ebenfalls, dass der Ruf der Tiroler Landesschützen als Eliteregimenter zur Folge hatte, dass diese sich bereits Ende des 19. Jahrhunderts fast nur mehr aus Freiwilligen zusammensetzten. Vgl. Lichem 1977, S. $13,29,39$.

51 Der Stern vom 10.06.1999, S. 152-158 („Der Erinnerung eine Zukunft geben“), S. 156, zitiert nach Kühne 2006, S. 11.

52 Allgemeine Dienstvorschrift für das Bundesheer (ADV). Online: http://www.bmlv. gv.at/pdf_pool/gesetze/wg2001.pdf [2012-09-20]. S. 50-51.

53 Vgl. Bernsdorf 1969.

54 Der Begriff „Kameradschaft“ leitet sich ab vom lateinischen „camera“, das bedeutet „Gewölbe, Raum mit gewölbter Decke“, und vom italienischen „camerata“, was „Stubengemeinschaft“ meint. Zunächst wird der Begriff von italienischen Söldnern im 
schen „Freundschaft“, die auf „persönlicher Sympathie, persönlichem Gleichklang oder wechselseitiger Anziehung“ beruht, und „Kameradschaft“, die in der "Gleichheit des Tuns und des Meinens, in der Gemeinsamkeit der Aufgaben“"55 besteht. Idealtypisch gedacht, werden, so Bernsdorf, persönliche Fähigkeiten ,im wesentlichen, unabhängig von persönlicher Sympathie oder Antipathie, im Hinblick auf ihren Wert für die Aufgaben der Kameradschaft eingeschätzt ${ }^{\text {“56 }}$, auch wenn die Grenze zwischen Freundschaft und Kameradschaft nicht deutlich gezogen werden könne. Persönliche Freundschaft könne zur Kameradschaft in Gruppen hinzukommen, sie kann aber auch fehlen. ${ }^{57}$ Insbesondere in formellen Organisationen wie beispielsweise dem Militär und in Gefahrensituationen lassen sich oftmals eine besonders enge Kameradschaft und große Gruppenkohäsion beobachten. ${ }^{58}$ Thomas Kühne, der das Phänomen „Kameradschaft" im Hinblick auf die deutschen Wehrmachtsoldaten im Zweiten Weltkrieg untersuchte, zeichnet bereits am Beginn seines Buches ein ambivalentes Bild von Kameradschaft: Zunächst scheint „Kameradschaft“ als das „Leitmotiv der Kriegserinnerungen der alten Soldaten in Deutschland“, bei näherer Betrachtung zeige sich aber, dass „die Kriegsgeneration keineswegs so homogene Erinnerungen an den Krieg und speziell an die Kameradschaft hat ${ }^{\text {“59 }}$. Was zumeist von den Soldaten unter Kameradschaft verstanden wird, sind stabile, auf Vertrauen basierende, persönliche Beziehungen in kleineren militärischen Einheiten, doch „gab es zu keinem Zeitpunkt“, wie Kühne in seiner Analyse der Kameradschaft der „Soldaten des nationalsozialistischen Krieges“ zeigt, „nur ein Verständnis von Kameradschaft, sondern viele, und diese Vielfalt war dem historischen Wandel unterworfen ${ }^{\star 60}$. Es stelle sich also die Frage, ,,was ,bedeutete“ Kameradschaft wann und für wen?“61 Während in offiziellen und semi-offiziellen Darstellungen „Kameradschaft" als eine der Tugenden von Soldaten eingefordert, beschworen und

16. Jahrhundert verwendet, alsbald findet er aber Eingang in andere europäische Sprachen. Vgl. Sorg 2004, S. 58.

55 Bernsdorf 1969, S. 527.

56 Ebd., S. 528.

57 Unterschiedliche Autoren versuchen differente Typen von sozialen oder persönlichen Beziehungen zu klassifizieren. Man denke in diesem Kontext etwa an Argyle und Henderson, die im Hinblick auf deren Merkmale vier Dimensionen: eng-oberflächlich, freundschaftlich-feindselig, egalitär-hierarchisch und aufgabenorientiert-gesellig, festmachen. Dabei können sich Beziehungen im Zeitverlauf in Bezug auf die Merkmalsausprägungen verändern. Vgl. Heidbrink / Lück / Schmidtmann 2009, S. 13-14.

58 Vgl. Bernsdorf 1969, S. 528.

59 Kühne 2004, S. 11.

60 Ebd., S. 19.

61 Ebd., S. 20. 
mitunter verherrlicht wird, ${ }^{62}$ zeichnen andere Quellen, wie unter anderem Autobiographien und Romane, ein differenzierteres Bild von Kameradschaft. ${ }^{63}$

Betrachtet man „Kameradschaft“ in theoretischer Hinsicht und mit Hilfe historischer sowie soziologischer Studien und Analysen also näher, so liegt die Vermutung nahe, dass verschiedene Emotionen wie beispielsweise „Vertrauen“, „Liebe, Zuneigung, Bindungsgefühl“, gemeinsam geteilte „Angst“ oder „Stolz“ und „Scham“ bei der Genese und Wirkungsmächtigkeit von „Kameradschaft" eine bedeutende Rolle spielen. Hinter „Kameradschaft“ steht also ein Gemenge verschiedener Gefühlslagen. ${ }^{64}$ Die von mir analysierten Quellen - Regimentsgeschichten, autobiographischen Manuskripte und Tagebücher - zeigen im Hinblick auf diese Gefühlslagen folgendes Ergebnis: $:^{65}$

In den Regimentsgeschichten ${ }^{66}$ wird vorrangig das Bild einer durch Vertrauensbeziehungen zusammengehaltenen homogenen Gruppe gezeichnet, die sich vom Regimentskommandanten bis zum einfachen Soldaten erstreckt. So um-

62 Vgl. dazu u.a. Lichem 1977 sowie Fröhlich 1937 und 1937a.

63 Mit dem Kameradschaftsbegriff verbunden ist oftmals der der Loyalität, die im Krieg die wichtigste zentrale Emotionen betreffende Tugend neben Tapferkeit und Disziplin darstellt. Loyalität und Treue wiederum werden oft in ähnlichen Kontexten beziehungsweise synonym zueinander verwendet. Haslinger unterscheidet im Hinblick auf „Loyalität“ drei, ineinander übergehende Komponenten: „,erstens eine emotionellethische Komponente, die individuelle und kollektive Handlungsdispositionen erfasst und dabei die Gegenseitigkeit der Beziehungen ins Zentrum der Überlegungen stellt; zweitens einen Verhaltensbezug, der für Akteure und Beobachter Zustimmungsleistung darstellbar, abrufbar, überprüfbar und sanktionierbar macht und damit Nachahmungsund Disziplinierungseffekte erzeugt; und schließlich drittens eine diskursive Komponente, die es Gruppen ermöglicht, über scheinbar festgefügte Identitätspositionen hinaus Handlungsspielräume festzulegen, innerhalb derer Allianzen, Zuordnungen und Abgrenzungen ermöglicht werden, die sich aus den Identitätspositionen allein nicht erklären ließen.“ (Haslinger 2007, 2) Haslinger geht dabei von einer Dualität des Loyalitätsbegriffs aus und unterscheidet demgemäß zwischen einer Innenperspektive, die die Einstellung bzw. Zustimmungshaltung des jeweiligen Akteurs umfasst, und einer Außenperspektive, die die Wahrnehmung konkreter Handlungen, wie die Teilnahme an Wahlen oder Festen oder die Folgeleistung eines Einberufungsbefehls, beinhaltet (vgl. ebd., 4). 64 Vgl. Haring 2013, S. 290-297.

65 Regimentsgeschichten wurden wie viele Autobiographien häufig mit einigem zeitlichen Abstand zum Kriegserlebnis verfasst. Die Regimentsgeschichten wurden dahingehend ausgewählt, als sie unter anderem auch jene Frontabschnitte und im Idealfall auch jene Regimenter beschreiben, in denen die Verfasser der ausgewählten Selbstzeugnisse dienten und/oder die darin ausführlicher behandelt wurden.

66 Als Quellen dienten in diesem Zusammenhang: Die Geschichte des steirischen k.u.k. Infanterieregimentes Nr. 27 (Fröhlich 1937, und Fröhlich 1937a) sowie die von Heinz Lichem von Löwenbourg unter dem Pseudonym Heinz von Lichem verfasste Regimentsgeschichte Spielhahnstoß und Edelweiß (Lichem 1977). 
schließt die Gruppensolidarität beispielsweise in der von mir untersuchten Regimentsgeschichte von Hermann Fröhlich ${ }^{67}$ den ,allbeliebten, hochverehrten“ Regimentskommandanten Karl Weber, die durchgehend als „tapfer“, „schneidig" und „opfermutig“ beschriebenen Frontoffiziere und die einfachen Soldaten. Die Teilung des Regimentsverbandes wie beispielsweise im Mai 1915 wird als besonders belastend beschrieben: „Die erste, wiewohl unblutige Einbuße wirkte sich in der Zerreißung des festgefügten Regimentsverbandes aus. Was dies bedeutet, vermögen nur der erfahrene Truppenoffizier, der schlichte Fußsoldat, der vielgeplagte Proviantoffizier voll zu erkennen." ${ }^{\text {68 }}$ Gegenseitiges Vertrauen und das Gefühl der Sicherheit werden - vor allem in Fröhlichs Regimentsgeschichte immer wieder thematisiert. Auch wenn die zahlreichen Fluktuationen in Folge von Ab- und Neuzugängen zu spürbaren Veränderungen in der Gruppenstruktur führen, scheint, folgt man der Deutung der untersuchten Regimentsgeschichten, die Gruppenkohäsion innerhalb kürzester Zeit wieder hergestellt. Wir-Gefühle werden vorrangig mit dem Begriff „Kameradschaft“ beschrieben. Bei Fröhlich gibt es vorrangig sehr indirekte Hinweise, was denn Kameradschaft ausmachen könnte: „Treue Kameraden“ helfen den Verwundeten und bleiben bei den Schwerverletzten. ${ }^{69}$ Besonders schmerzlich sei es, wenn die verwundeten Kameraden - so aus den vorderen Kavernen am Monte Forno - nicht zurückgebracht werden können. ${ }^{70}$ Der „letzte Kameradschaftsdienst“ ist schließlich, die Toten zu bergen und zu beerdigen. ${ }^{71}$ Kann man den Dienst, die Toten zu bergen, nicht erfüllen, bedrückt dies die Soldaten. ${ }^{72}$ Der Schutz anderer Kameraden beim Rückzug ist ein weiterer wichtiger Kameradschaftsdienst; Kameraden helfen auch den „Trennungsschmerz" von den Lieben in der Heimat abzumildern. ${ }^{73}$

Im Hochgebirge wiederum nehmen die Beziehungen der Soldaten zueinander, wie Lichem in seiner Regimentsgeschichte der Tiroler Landesschützen ${ }^{74}$, bei

67 Die Geschichte des steirischen k.u.k. Infanterieregimentes Nr. 27 wurde von Oberst d. R. Hermann Fröhlich, die Auszeichnungs- und Verluststatistik von Oberst d. R. Franz Rech Edler von Feleky und die Skizzen von Oberstleutnant i.d. R. Arnold Philipp verfasst; erschienen ist die Regimentsgeschichte im Jahre 1937 in zwei Bänden. Hermann Fröhlich wurde am 22. Mai 1876 in Wien als Sohn eines Volksschuldirektors geboren. 1894 trat er in die Theresianische Militärakademie in Wiener Neustadt ein, am 18. August 1897 erfolgte die Ausmusterung. Zunächst diente er beim Infanterieregiment 98, ab 1908 dann bei den 27ern. Seine aktive Laufbahn beendete Fröhlich Anfang Mai 1920 (am 1. Mai ging er in den Ruhestand). Er lebte in Graz, wo er 1954 auch verstarb.

68 Fröhlich 1937, S. 300.

69 Vgl. Fröhlich 1937, S. 35, 249, 460, 506 sowie Fröhlich 1937a, S. 173, 240.

70 Vgl. Fröhlich 1937a, S. 211.

71 Vgl. Fröhlich 1937, S. 72, 503.

72 Vgl. Fröhlich 1937, S. 309.

73 Vgl. Fröhlich 1937a, S. 128.

74 Die von Heinz Lichem von Löwenbourg unter dem Pseudonym Heinz von Lichem verfasste Regimentsgeschichte Spielhahnsto $\beta$ und Edelwei $\beta$ wurde genau 40 Jahre nach 
welchen der auch später vorgestellte Gottlieb Pomberger diente, deutlich machen möchte, eine besondere Form an. Denn der Soldat im Hochgebirge habe „Kameradschaft“ neben den „Tugenden“ der „Ritterlichkeit“, der ,körperlichen Zähigkeit" und der „geistigen Wendigkeit" erlernt und diese Tugenden von Generation zu Generation weitergegeben. Gemeinsames Handeln, Vertrauen und Solidarität kennzeichnen die Beziehungen innerhalb der jeweiligen Einheiten, wobei eine der Besonderheiten der hochalpinen Elitetruppe nach Lichem in den abgeflachten Hierarchien liegt, die sich aus der Besonderheit des alpinen Geländes ergeben. Einfache Soldaten und Offiziere kamen häufig aus demselben Bergdorf, sie waren Schulkameraden, Nachbarskinder oder Söhne alter Bergbauerndynastien. ${ }^{75}$ In Lichems Verständnis von Kameradschaft sind Vertrauen und bisweilen Freundschaft enthalten, die Kameraden sind durch gemeinsam geteilte Werthaltungen, durch geringe Standesdünkel und abgeflachte Hierarchien ,zusammenschweißt“. Doch inkludiert die Gemeinschaft nicht nur die „Kameraden“ - vom einfachen Soldaten bis zu den Offizieren - sondern letztlich auch den Kaiser: „Der Kaiser und seine Soldaten waren eine unangreifbare Welt für sich - eine Welt der grenzenlosen Ehre und Anständigkeit. “76 Lichem zeichnet wie Fröhlich ein homogenes Bild von Kameradschaft, die sich bisweilen auch auf andere Regimenter, auf die Trains, die den Nachschub regeln, sowie auf den deutschen Verbündeten erstreckt. ${ }^{77}$ Mit unterschiedlichen Perspektiven und Nuancen aus der Sicht der beteiligten Akteure und den damit korrespondierenden jeweiligen Gefühlslagen werden der Leser und die Leserin nicht konfrontiert.

Fröhlichs Regimentsgeschichte publiziert und zeichnet die Geschichte der Tiroler Hochgebirgstruppe „Die Kaiserschützen“von ihren Anfängen bis 1918 nach. Der 1941 in Graz geborene und 2007 in München verstorbene Militärhistoriker, Schriftsteller und Journalist Lichem befasste sich jahrzehntelang mit dem Ersten Weltkrieg im Hochgebirge sowie mit dem Thema Fotografie. 1982 promovierte er mit einer Abhandlung über die Tiroler Kaiserschützen. Im Zuge seiner Recherchen interviewte Lichem zahlreiche Überlebende, ging als Alpengeograph und Militärwissenschaftler alle hochalpinen Fronten der österreichisch-ungarischen und italienischen Hauptkampflinien ab und legte ein bekanntes Gebirgskriegsarchiv mit über tausend Original-Kriegsakten, Tagebüchern, Kriegslandkarten und Originalfotografien an. Darüber hinaus war Lichem am Aufbau des bekannten, die Isonzofront darstellenden Kobariski Muzej in Kobarid (Slowenien) beteiligt, dem er zahlreiche Unterlagen aus seinem Militärarchiv zur Verfügung stellte. Für diese Mitarbeit wurde er mehrfach ausgezeichnet. Mehr als zehn Jahre widmete sich Lichem, wie er im Vorwort darlegt, sowohl beruflich als auch privat „mit größter Passion“ der Geschichte der k.k. Landesschützen-Kaiserschützen-Regimenter I, II und III mit der Intention, ,jenen, die mit Stolz den Spielhahnstoß und das Edelweiß führten, Gerechtigkeit und Ehre widerfahren zu lassen: Den lebenden und toten Landesschützen-Kaiserschützen!“ (Lichem 1977, S. 10)

75 Vgl. Lichem 1977, S. 30.

76 Lichem 1977, S. 11.

77 Vgl. Haring 2013, S. 380-385 sowie S. 388-392. 
Offiziere und Soldaten scheinen dem moralischen Code der Kameradschaft, folgt man den Regimentsgeschichten, zu entsprechen, ja ihn als eine Selbstverständlichkeit verinnerlicht zu haben.

Eine teilweise andere Erfahrung von Kameradschaft an der Front und in der Etappe beschreiben die von mir analysierten Ego-Dokumente. Das Bild eines homogenen Ganzen von Offizieren und Mannschaften und auch das der Einheit von Soldaten innerhalb der Mannschaften sind in den untersuchten Tagebüchern und Autobiographien nicht zu finden. ${ }^{78}$ Das Verhältnis zu Offizieren ist ambivalent - bisweilen sind die eigenen Offiziere die „Feinde“ der einfachen Soldaten. Das bedeutet, dass diese den Offizieren häufig Gefühle entgegenbringen, die man gemeinhin mit Emotionen gegenüber „Feinden“ assoziiert. Die soziale Distanz der einfachen Soldaten zu den Offizieren ist groß, das Verhältnis zu ihnen, aber auch zu gleichrangigen Kameraden oft angespannt. ${ }^{79}$ Man ekelt sich vor einander, insbesondere gegen die Offiziere richtet sich oft ohnmächtige Wut. Bisweilen hasst man diese auch. Ein besonders negatives Bild im Hinblick auf „Kameradschaft“ zwischen Offizieren und Mannschaften, also im Hinblick auf „,vertikale Loyalität“, zeichnet beispielweise das Tagebuch von Franz Matthias Hartinger, in dem dieser immer wieder Züchtigungen und das Anbinden von Soldaten erwähnt:

Was sich dieser Herr an Rohheiten leistet, verdient angenagelt zu werden. Es gibt hiefür keine Entschuldigungen, weder Nervosität noch ein anderes Leiden. Feldwebel werden geschlagen, dass ihnen das Blut herunterrinnt, die gemeinsten Schimpfworte fliegen wie die Kugeln auf Podgora herum. Die grössten Leiden erduldet der arme Bursche. Ein williger, beschränkter Slowene. ${ }^{80}$

Die Soldaten gehen „zitternd“ zum Oberleutnant Hübner, Schlagen und Schimpfen seien an der Tagesordnung. Doch diejenigen, die die Pflicht hätten, diesen Brutalitäten Einhalt zu gebieten, schauen weg: ,Jeder schaut, Auszeichnungen zu bekommen, aber dass sich wer um die Mannschaft schert, das gibt es nicht. Ich liege auf einer Wiese und ärgere mich wie ein Frosch. “81 Kameradschaftliche Homogenität zwischen Offizieren und Mannschaften sowie ausschließlich „gute“ Vorgesetzte kann man in Hartingers Tagebuch ${ }^{82}$ nicht finden.

78 In diesem Zusammenhang wurden die autobiographischen Manuskripte von Gottlieb Pomberger, Franz Obergottsberger und Matthias Royer sowie das Tagebuch von Franz Matthias Hartinger untersucht.

79 Zu den Erfahrungen im Heer aus der Sicht ,einfacher Rekruten“ vgl. unter anderen Christa Hämmerles „Des Kaisers Knechte“ (2012).

80 Hartinger 2012, S. 126.

81 Hartinger 2012, S. 127.

82 Hartinger (1880-1951) war an verschiedenen Kriegsschauplätzen im Einsatz. Sein Enkelsohn Volker Rutte hat die Tagebücher, die er in einer roten Blechdose von „400 
Vielmehr werden diese oft als die „Feinde“ der einfachen Soldaten gezeichnet. ${ }^{83}$ Auch in dem autobiographischen Manuskript von Matthias Royer, einem Ramsauer Bauern, der am Kampf um die von den Russen belagerte Festung Przemyśl beteiligt war und nach der Übergabe der Festung in russische Kriegsgefangenschaft ging, findet sich diese negative Einschätzung der Offiziere wieder:

Jetzt [zur Zeit der zweiten Belagerung; Anm. d. Verf.] mußte die arme Mannschaft die Sorglosigkeit und die Pflichtvergessenheit ihrer Vorgesetzten auf schauderhafte Weise büsen, während die Herren Offiziere wie zuvor in Saus und Braus lebten, während von der armen Mannschaft, die noch mit Dienst überbürdet wurde und in der strengen Winterkälte keine warme Kleidung hatte, 13000 Mann an Hungertyphus starben. ${ }^{84}$

Die Kameraden - höhere Offiziere, Unteroffiziere und Mannschaften - erscheinen in den untersuchten Tagebüchern und auch in den analysierten autobiographischen Manuskripten nicht als ein homogenes Ganzes wie in den Regimentsgeschichten, sondern werden differenziert dargestellt. So wird der Einjährig-Freiwillige Franz Obergottsberger ${ }^{85}$ während seiner Zeit in der Reserve mit dem Einjährigen-Zugsführer Bakic in einem von ruthenischen Bauern - einer hochschwangeren Frau, einem vierzehnjährigen Mädchen und einem kleinen Buben - bewohnten Lehmhaus einquartiert. Obergottsberger zeigt - wie auch die

Gramm Dessert-Waffeln“ gefunden hatte, schließlich abgeschrieben, mit Erklärungen und Kommentaren versehen und dankenswerter Weise meinem Kollegen Helmut Kuzmics und mir für unsere Analysen zur Verfügung gestellt. Der Enkelsohn Volker Rutte charakterisiert Hartinger als ein „Grazer Original“ mit deutschnationaler Gesinnung. 83 Auch in dem autobiographischen Text von Gottlieb Pomberger erscheinen die Offiziere bisweilen als die „Anderen“, sie werden von ihm durchaus auch negativ geschildert. Sobald ein Sturmangriff der Feinde bevorsteht, bekommt man „keinen Offizier mehr zu Gesichte“, erst bei Eintreffen am Sammelplatz nach dem Gefecht treffe man sie wieder. Auch bei Franz Obergottsberger finden wir eine ambivalente Einschätzung der Offiziere. In der Ausbildungszeit marschiert er unter der Führung des Oberleutnants Enzinger und des Fähnrichs Lill als „Abrichter“ auf die „Schinderwiese“. Sympathie empfindet Obergottsberger Fähnrich Lill gegenüber, der „sich durch seine Jugend noch sehr" mit den Auszubildenden verbunden fühlt und deshalb, so Obergottsberger, zur „Güte“ neigt, während Oberleutnant Enzinger sich - obwohl er zur Reserve gehört - wie ein „,aktiver Offizier“ benimmt, keinen Spaß kennt und ohne Erbarmen agiert. Er legt für Obergottsberger ein anmaßendes Verhalten an den Tag; Abneigung ist hier deutlich zu spüren (vgl. Obergottsberger II o. J., S. 4-5).

84 Royer o. J., S. 12.

85 Franz Obergottsberger wurde 1895 als uneheliches Kind einer Dienstmagd geboren und ist in bescheidenen Verhältnissen bei seiner Großmutter aufgewachsen. Er absolvierte das Kollegium Petrinum in Linz. Schließlich meldet er sich als Einjährig-Freiwilliger zum Infanterieregiment Rainer Nr. 59 und bekommt am 26. Oktober 1915 den Bescheid, „behalten worden zu sein“. 
anderen Petriner - Verachtung jenen gegenüber, die nur vom „Vögeln“ reden. Insofern ist ihm auch das Zusammenleben mit Bakic unangenehm, der mit lüsternen Blicken die Bewegungen der schwangeren Frau verfolgt und dessen Gespräche sich nur um diese drehen. ${ }^{86}$ Das „derbe“ Verhalten ruft bei Obergottsberger Ekel und Verachtung hervor, also Emotionen, die man eher im Zusammenhang mit dem „Feind“ oder mit den „Fremden“ vermutet. Deutlich wird in den beiden autobiographischen Texten von Pomberger und Obergottsberger jedoch auch die Bedeutung von einzelnen Kameraden für ihren Affekthaushalt an der Front. In beiden Manuskripten werden „Freundschaften“ beschrieben, einzelne „Kameraden“ werden als „Freunde“ und/oder „Brüder“ bezeichnet. In beiden Kriegserzählungen werden Wir-Gefühle zu einzelnen Kameraden von Beginn an deutlich. Einzelne Soldaten - bei Pomberger meist aus Oberösterreich oder Niederösterreich - werden als „Freunde“, „Brüder“, als „gute und ehrliche Kameraden" bezeichnet. Die älteren Soldaten zeigen sich zunächst den jüngeren gegenüber „boshaft“ und „feindselig“, sie lassen sich vom Jüngeren, also von Pomberger, nichts sagen. Die Beziehung bessert sich - wie Pomberger anmerkt bei näherem Kennenlernen und wenn man die Spielregeln, die Codes, einhalte. ${ }^{87}$ Bei Obergottsberger sind es zunächst vor allem seine Petriner Kameraden, auf die sich Bindungsgefühle und Vertrauen erstrecken. Vertraute Gesichter erzeugen Freude und Glücksempfindungen. Bei einem Vorstoß Ende Oktober 1914 stirbt im „mörderischen Maschinengewehrfeuer“ Pombergers Freund Pitsche, Pombergers Stimmung erreicht einen Tiefpunkt, sie „trübte sich masslos, waren wir doch schon in Fiera di Primiero also in der Kaserne gute Freunde“ ${ }^{\text {(88 }}$.

\section{Feindbilder und Feindseligkeit}

Das Wir-Gefühl innerhalb der eigenen Gruppe wird durch Definitionen von outgroups und die Grenzziehung zwischen dem Eigenen und dem Fremden gestärkt. Das Feindbild wiederum schafft, wie Wagener unterstreicht, eine „Übereinstimmung ex negativo [...], , territorialen' Konsens auf Kosten Außenstehender“. ${ }^{89}$ „Feinde“" sind, so Zygmunt Bauman,

die Negativität, der gegenüber die Freunde das Positive darstellen. Die Feinde sind, was die Freunde nicht sind. Die Feinde sind „umgekehrte“ Freunde; sie sind der Dschungel, der die vertraute Ordnung der Freunde aufhebt; die Abwesenheit, die die Gegenwart des Freundes leugnet. Das widerwärtige und ängstigende „da Draußen“ der Feinde ist, wie

86 Vgl. Obergottsberger II o. J., S. 28.

87 Pomberger o.J., S. 5.

88 Pomberger o. J., S. 42.

89 Wagener 1999, S. 26. 
Wir-Gefühle, Feindbilder und Feindseligkeit bei deutschsprachigen k.u.k. Soldaten

Derrida sagen würde, ein Supplement: sowohl Ergänzung und Verdrängung des gemütlichen und komfortablen „Drinnen“ der Freunde. ${ }^{90}$

Definitionen von Eigenem und Fremden unterliegen dabei einem ständigen Wandel, sie sind äußerst komplex und teilweise ambivalent, manchmal auch mehrdeutig. Auf einer theoretischen Metaebene, wie sie von Wagener und Bauman eingenommen wird, erscheinen „Feinde“ im Unterschied zu Freunden als das absolut Negative, als das Böse sowie als das zu Bekämpfende. Sie sind dadurch gekennzeichnet, dass man für sie keine Verantwortung und Verpflichtung übernimmt. Ob sich diese Charakterisierungen von „Feinden“ auch in den von mir untersuchten Regimentsgeschichten, autobiographischen Manuskripten und Tagebüchern finden lassen, soll im Folgenden kaleidoskopartig gezeigt werden.

Im Hinblick auf Fremdheitsgefühle und Feindbilder gilt es in einer emotionssoziologischen Perspektive demnach zu fragen: Wen benennen die untersuchten Quellen als „Feinde“ oder „Fremde“ und welche Gefühle bringen sie zum Ausdruck, wenn sie von „Feinden“ sprechen? Welche Grenze wird zwischen dem „Wir" und den „Anderen“ gezogen? Welche Rolle spielen Emotionen wie Angst, Ärger, Wut, Zorn, Ekel, Abscheu, Verachtung und Hass, die man gemeinhin mit „Feinden“ verbindet, im Hinblick auf „Feinde“ an der Front, gegen die die k.u.k. Soldaten kämpfen, und im Hinblick auf „Fremde“, die ihnen an der Front, in der Etappe oder auch im unmittelbaren Hinterland begegnen ${ }^{91}$

Im Krieg gesellt sich neben die

Angst vor dem Feind, der das eigene Leben bedroht, [...] die Angst vor dem sozialen Tod durch Ausschluss oder gar dem physischen Tod durch die eigene Gruppe, wenn man den Anforderungen des Kollektivs nicht gerecht wird oder sich ihnen zu entziehen versucht. ${ }^{92}$

Ausgrenzung und Tod bei Desertion sind mögliche Folgen. Desertion galt als „Treuebruch“, der unterschiedlich - unter anderem mit Freiheitsentzug, Todesstrafe, Einzug des Vermögens bis hin zu „Höllenstrafen“, die von Militärgeistlichen den Soldaten angedroht wurden - sanktioniert worden ist. ${ }^{93}$

Als Fremde im Baumanschen Sinne, als Menschen, die sowohl Freund als auch Feind sein können, ${ }^{94}$ wird in den Regimentsgeschichten vor allem die Zivilbe-

90 Bauman 1998, S. 23.

91 Eine detaillierte Analyse dieser Emotionen findet sich bei Haring 2013.

92 Friedman 2015, S. 196.

93 Vgl. Buschmann / Murr 2008, S. 22.

94 Der berühmte Aufsatz von Bauman Moderne und Ambivalenz beginnt mit der Differenzierung von „Freunden“, „Feinden“ und „Fremden“: „Freunde und Feinde stehen in Opposition zueinander. [...] Gegen diesen vertrauten Antagonismus, dieses konflikthafte Einverständnis von Freunden und Feinden rebelliert der Fremde." (Bau- 
völkerung am russischen Kriegsschauplatz gezeichnet. ${ }^{95}$ Die Tatsache, dass die Soldaten nicht wissen, ob sie Freunden oder Feinden gegenüberstehen, erzeugt genau das Gefühl der Unsicherheit, das zu einem Bedrohungsgefühl wachsen kann, das Bauman beschreibt. Zivilisten und Zivilistinnen werden nicht nur als „Opfer“, sondern auch als „Täter“ als „Russophile“ eingeschätzt und als Spione gebrandmarkt, die ,kurzerhand kaltgemacht" werden $:^{96}$

Immer mehr verdichteten sich die Meldungen über verräterische Anzeichen in der zu einem nicht unerheblichen Teile russophil eingestellten Bevölkerung. Ein Gefühl der Unsicherheit überkam die Truppe, sie vermeinte sich schließlich überall von Verrat umlauert. Da und dort beschritt sie den Weg der Selbsthilfe. ${ }^{97}$

Die Mehrfachidentität der Zivilbevölkerung in multiethnischen Gebieten wurde als Mangel an Abgrenzung zum Feind interpretiert, als es bei Kriegsausbruch und im Verlauf des Krieges darum ging, ,klare Fronten“ zu schaffen. Verschärfend kam hinzu, dass die Grenzen der Habsburgermonarchie sich in Aufmarsch-, Gefechts- und Besatzungszonen auflösten, wobei insbesondere die ungarischen und aus dem heutigen Österreich stammenden Soldaten Gebiete an der Ost- und Balkanfront als „fremd“ empfanden. Strafen gegenüber den Fremden - dem „Kaltmachen“ oder „Niedermachen“ - geht in der Erzählung der Regimentsgeschichten immer eine Aktion der „Russophilen“ voraus - ein Tabu der Regimentsgeschichten bleibt, wie Wencke Meteling im Hinblick auf die deutsche Armee herausarbeitete, dass Soldaten Gewalt gegen Unschuldige verübten. Denn dies wäre mit dem Bild des Soldaten, der ehrenvoll und in Würde gegen den Feind kämpft, nicht in Einklang zu bringen. ${ }^{98}$ Folgendes Zitat aus der Regimentsgeschichte der 27er macht dies nochmals deutlich:

man 1998, S. 23, 25) Gelten, worauf Simmel hinwies, sowohl Freundschaft als auch Feindschaft als Grundmuster der Vergesellschaftung, „gefährdet", so Bauman in Moderne und Ambivalenz, „der Fremde das soziale Leben selbst. Und all dies, weil der Fremde weder Freund noch Feind ist; und weil er beides sein könnte“. (Bauman 1998, S. 25)

95 Auch in Österreich-Ungarn lässt sich am Beginn des 20. Jahrhunderts ein Prozess der Ethnisierung beobachten, der durch ein „Nebeneinander von ethnischer Neutralität, Anerkennungspolitik und Diskriminierungstendenzen“ (Benno Gammerl, zitiert nach Leidinger 2014, S. 56) gekennzeichnet war. Die Angst vor Verrat in den eigenen Reihen führte vor dem Hintergrund der Krisen vor 1914, wie Hannes Leidinger ausführt, zu einer regelrechten „Spionenmanie“, wobei besonderes Misstrauen ethnischen Minderheiten innerhalb der Habsburgermonarchie entgegengebracht wurde (vgl. Leidinger 2014).

96 Vgl. Fröhlich 1937, S. 334, 343, 346.

97 Fröhlich 1937, S. 41.

98 Vgl. Meteling 2010, S. 218. 
Untrügliche Anzeichen von Verrat und Spionage veranlaßten das Bataillonskommando, die Evakuierung des Ortes zu beantragen. Das 43. LIBrigKmdo. [Landwehr-Infanteriebrigadekommando; Anm. d. Verf.] verfügte die sofortige Aushebung von 31 Geißeln, die in der Ortskirche von Olchowiec interniert wurden. Die Evakuierung ging in der Nacht auf den 14. vonstatten. ${ }^{99}$

Insbesondere der Vormarsch in fremdes Territorium verschärfte die Lage:

Der Bewegungskrieg - und nicht so sehr der Stellungskrieg - wirkte brutalisierend. Die Angst vor dem Fremden grassierte speziell in ,asymmetrischen“ Konflikten, bei Konfrontationen ungleicher Kontrahenten, die vorwiegend unterlegene Streitparteien bisweilen dazu zwangen, sich unkonventioneller Kampfformen zu bedienen. ${ }^{100}$

In Pombergers autobiographischem Manuskript finden sich mehrere Passagen zur Zivilbevölkerung, die zwar als die Anderen im Sinne von Nicht-Soldaten erscheint, für die Pomberger jedoch meistens Mitleid zum Ausdruck bringt, insbesondere dann, wenn die Menschen mit wenigen Habseligkeiten gezwungen sind $\mathrm{zu}$ flüchten und schwache und kranke Familienmitglieder zurücklassen müssen:

Welchen Schmerz diese Leute beim Verlassen ihrer heimatlichen Schollen unter solchen Verhältnissen gehabt haben mögen, ist erklärlich, wenn man sich vor Augen hält, dass nicht nur alle Einrichtung und der Viehbestand, sondern oftmals auch alte gebrechliche Leute im Stiche gelassen werden mussten. Dies alles für Gott, Kaiser und Vaterland $!^{101}$

Die Häuser der Menschen werden von beiden Artillerien beschossen, die auf den Wiesen frei herumlaufenden Rinder und Schweine werden „oftmals unter schauderhaftem Gebrüll“ zu „Opfern der Schrapnells“. Die Häuser brennen restlos ab. ${ }^{102}$ Ähnlich schildert Matthias Royer die Lage der Zivilbevölkerung rund um Przemyśl:

Als unsere Feldarmee den Rückzug antreten musste, da wurden im Vorfeld in der Umgebung von Przemysl alle Dörfer von deren Bewohnern gesäubert. Die mußten in langen, traurigen Zügen ihre Heimat mit Weib und Kind und alten Personen verlassen, die Männer waren so die meisten im Feld und wurden unter Bewachung nach Österreich geliefert. Kaum hatten die Bewohner die liebe, teure Heimat verlassen, so wurde das Dorf angezündet. Fast jede Nacht brannte ein solches Dorf [...]. Die Dörfer wurden aber

99 Fröhlich 1937, S. 353.

100 Leidinger 2014, S. 69. - Nicht zuletzt aufgrund zahlreicher Denunziationen wurden sogenannte Proskriptionslisten angelegt, auf welchen nicht selten ganze Ortschaften der Spionage und des Verrats bezichtigt wurden (vgl. S. 82-83).

101 Pomberger o. J., S. 52.

102 Pomberger o. J., S. 52. 
samt den darin befindlichen Getreidevorräten, welche wir so notwendig gebraucht hatten und viel vorhanden sein mußten, verbrannt. ${ }^{103}$

Die „Fremden“ sind aber nicht nur die in den Kampfgebieten lebende Zivilbevölkerung, sondern teilweise auch die Kameraden anderer Regimenter und ethnischer Zugehörigkeiten, denen teilweise „mangelnde Tapferkeit“ oder „Zuverlässigkeit“ zugeschrieben wird und die man insofern als „schlechte Kameraden“ betrachtet, oder die man - so Obergottsberger - für ihr ,vulgäres Verhalten“ und ihre „Trunksucht“ verachtet. Das Gefühl des Fremdseins wird durch sprachliche Barrieren noch verstärkt. Immer wieder werden in autobiographischen Dokumenten Sprachbarrieren zwischen den Soldaten erwähnt, die die Kommunikation erschwerten. ${ }^{104}$ Der Fremde wird schließlich beim „Verrat“ vor allem in den Regimentsgeschichten zum „Feind“, der „Verrat“ bedeutet hier den „Treuebruch“. ${ }^{105}$ So schildert Fröhlich, auch unter Bezugnahme auf die Geschichte des Infanterieregiments Nr. 47 ${ }^{106}$, die Ereignisse rund um den „Verrat" der 28er, welche ausführlich von Richard Lein in Pflichterfüllung oder Hochverrat? dargestellt wurden. ${ }^{107}$ Lein, der den „Fall“ des Infanterieregiments

\section{Royer o. J., S. 10.}

104 Vgl. Grünwald / Hauer / Huber / Stummer 2009/2010, S. 229.

105 An einer Stelle der Regimentsgeschichte berichtet Lichem vom „wohl krassesten Fall von Verrat bei einem österreich.-ung. [österreichisch-ungarischen; Anm. d. Verf.] Truppenkörper an der Italienfront“ - dem „Verrat von Carzano“. Hier findet sich der „Feind“ also innerhalb der eigenen Truppe. Unter der Führung des Oberleutnants Pivko aus Marburg hätten tschechische und slowakische Soldaten italienische Angriffstruppen - teilweise in k.u.k. Uniformen - durch die eigenen Linien geführt, Losungswörter weitergegeben, die Stromsperren in den eigenen Drahthindernissen ausgeschaltet et cetera. (Vgl. Lichem 1977, 205; zum „schimpflichen Verrat von Carzano“ auch Fröhlich 1937a, 228 f.)

106 Vgl. Vogelsang 1932; Vogelsang 1932a.

107 Das Bild vom „unwilligen“ und „untreuen“ tschechischen k.u.k. Soldaten war nicht nur in der Ersten Republik und in der Weimarer Republik, sondern auch in der neugegründeten Tschechoslowakei stark verbreitet. Während die bereits während des Krieges von Deutschnationalen vertretene Sicht der Unzuverlässigkeit der Tschechen als eine „österreichische Variante der reichsdeutschen Dolchstoßlegende“ (Ivan Šedivý nach Lein 2011, S. 12) interpretiert werden kann, wurden die vermeintlich zu Massen desertierten Soldaten zunächst von der tschechischen Exilregierung und später in der Tschechoslowakei als Helden gefeiert, die die Gründung des neuen Staates erst möglich gemacht hätten. Sie galten bereits während des Krieges den Ententemächten als Beleg für die Existenz eines tschechischen Widerstandes. Das „Verhalten der tschechischen Soldaten Österreich-Ungarns im Ersten Weltkrieg gehört“, wie Lein unterstreicht, ,zu den umstrittensten Themen der jüngeren Geschichte Ostmitteleuropas“ (Lein 2011, S. 7). Im Heldendiskurs der Zwischenkriegszeit galten in der Tschechoslowakei neben den 
Nr. 28 in der Schlacht bei Esztebnekhuta 1915 und den der Infanterieregimenter 35 und 75 in der Schlacht bei Zborów 1917 untersucht, kommt dabei im Hinblick auf die von Fröhlich und vielen anderen Autoren geschilderte Verratsepisode des Prager Hausregiments, des Infanterieregiments 28, im Jahre $1915 \mathrm{zu}$ folgendem Schluss: Weder habe ein Verrat noch ein Überlaufen der k.u.k. Soldaten tschechischer Herkunft stattgefunden.

Selbstredend gab es Beispiele von Selbstverstümmelung und Desertion in der k.u.k. Armee: So berichtet Hartinger in seinem Tagebuch, dass im Oktober 1916 mehrere Kompanien „übergegangen“ seien: „Ja, wir alpenländischen Wurzen. Czechen unzuverlässig, Ungarn nur mit dem Maul, Rumänen gehen über. “ ${ }^{\text {"108 }}$ Dass der Desertion oftmals ein schweres Ringen mit sich selbst vorausging, macht insbesondere der slowenische Antikriegsroman Doberdó deutlich: Als die Festung Przemyśl, das „,Tor zu Ungarn“, fällt, fühlt sich der slowenische Soldat Amun und mit ihm viele andere gespalten: „In seinem Kopf“", so Amun,

und in seinem Herzen wallten so viele Gedanken und so viele Gefühle auf, dass er noch nicht klar sah, was er denken und wie er urteilen sollte. Er stand wie an einem Kreuzweg. Auf der einen Seite jauchzte sein Herz vor stiller Freude, dass es den Russen gelungen war, die Festung einzunehmen, die auch seiner Meinung nach dem russischen Vordringen nach Westen bisher den Weg versperrt hatte, einem Vordringen, das für die slawischen Völker die Freiheit bringen konnte; auf der anderen Seite hinderte ihn etwas daran, was er sich nicht erklären konnte. Er spürte ein seltsames Brennen bei den Gedanken, dass das zusammenbrach, dem er selbst angehörte. ${ }^{109}$

Nachdem Amun nach mehreren Wochen in den Gräben im Frühjahr 1916 unversehrt zurückgekehrt ist, reift - nach intensivem Abwägen und Gesprächen mit etwaigen Gesinnungsgenossen - in ihm endgültig der Entschluss, allein zu desertieren, auch wenn er sich dabei gleichzeitig einsam fühlt:

Die Italiener waren zwar die Gegner dessen, was ihm lieb und teuer war, aber sollte er einen Gegner gegen den anderen verteidigen? [...] Er verriet niemanden, weil man seinen Gegner nicht verraten konnte. Man kann allerdings sich selbst verraten, indem man diesem Gegner diente. ${ }^{110}$

Legionären auch die angeblich Übergelaufenen als Helden des Krieges (vgl. Stegmann 2010).

108 Hartinger 2012, S. 105. - Im November 1916 hält Hartinger die Zusammensetzung des Landsturm-Infanterieregiments 27 wie folgt fest: „Nationalitäten: Deutsche $19 \%$, Slovenen $41 \%$, Czechen $14 \%$, Polen $14 \%$, Kroaten $7 \%$, Italiener $1 \%$, Ruthenen 3,6 \% , Juden 0,5 \%.“ (Hartinger 2012, S. 110)

109 Kuhar 2008/2009, S. 47. - Zur detaillierten Analyse von Doberdó unter emotionssoziologischem Fokus vgl. Haring 2014.

110 Ebd., S. 201. 
Emotionen wie Ekel, Verachtung, Wut und Hass, durch die unter anderem Feindschaft charakterisiert wird, richten sich - außer gegen schikanöse Vorgesetzte - in den untersuchten Quellen gegen ganz unterschiedliche Personengruppen. Selbstredend werden die Russen und die Italiener, gegen die man kämpft, als „Feinde“ bezeichnet. Vor allem auf der Makro-Ebene, dort wo das politische und militärische Geschehen von Fröhlich, aber auch von Pomberger kommentiert wird, bedienen sich die Schreiber propagandistischer Stereotype, es lässt sich in manchen Passagen „Hass“ und „Verachtung“ nachweisen. In der direkten Konfrontation mit dem Gegner wird der „Feind“ jedoch als „ebenbürtig" beschrieben und ihm - sowohl den Russen als auch den Italienern - unterschiedliche, für das „Kriegswesen positive“ Eigenschaften zugeschrieben; Respekt zeigt sich. (Nur Pomberger spricht im Hinblick auf die Russen von „mangelndem Mut“.)

Dieser Respekt bedeutet jedoch nicht, dass man im konkreten Kriegsgeschehen nicht „wutentbrannt" gegen die Feinde vorgeht und sie „nieder-“ und „kaltmacht": Der Respekt schlägt also bisweilen in wilde „Angriffslust“ um, in folgendem Beispiel durch den Tod von Kameraden ausgelöst. Beim Vormarsch trifft man auf eine Schwarmlinie:

Der Sensenmann hatte ihr [der Schwarmlinie von 10 bis 15 Mann; Anm. d. Verf.] alles Leben vertilgt. Nur einige Schwerverwundete wälzen sich in den Ackerfurchen. Doch jetzt heißt es hart bleiben! Zum hilfreichen Kameradendienste sind andere ausersehen. „Vorwärts! An uns liegt es, die armen Dulder zu rächen!“‘111

Das Elend der Kameraden soll unmittelbar gesühnt werden. Doch schlägt die Rache wenig später wieder in Mitleid mit dem Feind um: Nach dem Kampf um das Dorf Zuszyce bot der „Hohlweg [...] ein grauenvolles Bild der Verwüstung: viele tote und verwundete Russen, schreiend, jammernd, nach Hilfe rufend“"112. Dem Kommandanten des III. Bataillons Hauptmann Anton Schwarz bietet sich ein ,gräßliches Bild“:

Es schien, als hätte die Totenhand plötzlich den Kampf unterbrochen. Zuerst glaubte ich unsere Schwarmlinie im Kampfe eingeschlafen, dann sah ich erst, daß es russische Schwarmlinien waren, geschlossene Feuerlinien, ein Russe neben dem anderen, die gutmütigen Gesichter bleich, aber keineswegs entstellt. Der rechte Zeigefinger noch am Abzuge oder gerade in der Ladestellung. Neben derben Bauerngestalten sah ich zarte, junge Kinder der Städte mit feinen arbeitsungewohnten Händen. Das Maschinengewehrfeuer hatte sie alle niedergemacht. ${ }^{113}$

111 Fröhlich 1937, S. 63.

112 Fröhlich 1937, S. 67.

113 Fröhlich 1937, S. 71. 
Bei einem Sturmangriff am 9. September 1914 - („Feuertaufe der IIIer Landesschützen bei Lelechowka) erlebt Gottlieb Pomberger das dem Kodex von Ehre widersprechende und aus der Sicht der Landesschützen zu verurteilende Verhalten:

Ehe wir den Wald vollkommen verlassen hatten, vereinigten wir uns mit unserem dritten Bataillon und gingen mit diesem - nachdem das Bajonett ,gepflanzt" war, im Sturme vor. Die Russen feuerten verzweifelt. Das konnte uns aber in unserer Erbitterung nicht mehr beirren, wir gaben auf keinen Fall mehr nach und so mussten die Russen unter $\mathrm{Zu}$ rücklassung einer Menge Gefangener das Feld räumen. Der Umstand, dass der Feind gleich nach Lautwerden unserer ersten Hurrarufe, das Zeichen des Ergebens hisste, trotzdem aber ein Feuer fürchterlichen Umfanges auf uns eröffnete, brachte uns in masslose Wut, umsomehr, als wir vielleicht ein Viertel unserer Mannschaft verloren hatten. ${ }^{114}$

Das, was folgt, ist ein „schreckliches Gemetzel“, dem auch Johann Scheffenbichler (Schatterhans) aus Russbach, der dem dritten Bataillon angehörte, „beiwohnte" - oder an dem dieser sich wohl wie Pomberger selbst beteiligte. In Wut und geleitet von Rachegefühlen werden die Russen - ohne Befehl und Beteiligung der Offiziere - verfolgt, dem schwer verwundeten russischen Kommandanten ein Schluck Wasser verweigert: „In unserer Erbitterung aber, hatten wir für ihn kein Wasser, sondern nur Kolbenstösse übrig. Eine solche Rohheit vollführt zu haben ist wohl grausam und kann nur in unbegrenztem Zorn geschehen. "115 Pomberger schildert also nicht nur das „unehrenhafte“ Verhalten der Russen, sondern auch die Reaktion der Landesschützen, die sich von Wut und Zorn getrieben, brutal rächen und somit ebenfalls gegen den Kodex der Ehre verstoßen - und das „ohne Befehl“ und folglich auch „ohne Mitwirkung“ der Offiziere. ${ }^{116}$ Hier wird die eigene Gewaltausübung als auch jene der Kameraden als „überzogen“ angesehen. Im Antikriegsroman Doberdó von Lovro Kuhar findet sich eine ebenso eindrucksvolle Schilderung: Nachdem die Protagonisten in Kuhars Roman sich für einige Wochen in einem Dorf von den Strapazen des Kampfeinsatzes erholt haben, werden sie an einem relativ ruhigen Frontabschnitt, in einer Senke zwischen der Anhöhe des San Martino und dem Monte San Michele, eingesetzt. Sich hinter Palirs Schießscharte verschanzend, schießt Almer im Scharfschützenstil auf italienische Soldaten:

Er [Almer] hockte dort wie einbetoniert und wartete besessen auf seine Opfer. Obwohl es nicht heiß war, lief ihm der Schweiß über den angespannten Nacken. [...] Almer wurde von wahrer Leidenschaft gepackt. So könnte man schön versteckt hinter einer sicheren Schießscharte sitzen und in Abständen den Abzug ziehen, neue Patronen durchladen,

114 Pomberger o. J., S. 24.

115 Ebd., S. 25.

116 Vgl. ebd., S. 26. 
und dort, hundert Meter weiter, würde es einen Feind nach dem anderen auf die Nase hauen. Auf diese Art könnte man in der Stunde sagen wir fünfzehn liquidieren, am Tag hundertsechzig, in der Woche ... ${ }^{117}$

Dieser Form von „Heldentum“ können viele der Kameraden nichts abgewinnen, weder die Neuzugänge, noch die Alten, die Almers Zielschießen als Unrecht empfinden: „Menschen waren doch keine Karnickel, die man einfach so abknallen konnte! Auch wenn es Italiener waren. Das war schon kein Krieg mehr..."118

Der kämpfende Italiener oder Russe gehört hier dem „Wir“ im Sinne einer „Schicksalsgemeinschaft“ als „Frontgemeinschaft" an. Die „Anderen“, die Feinde, die man verachtet und manchmal auch hasst, sind - neben einzelnen Kameraden der eigenen Einheit oder den vorgesetzten Offizieren - dann die Etappisten, die höheren Führungsebenen, die das „Vertrauen“ in die Truppen verloren hätten, oder die Hetzpropaganda im Hinterland. So hält Hartinger in seinem Tagebuch fest:

28. Jänner 1917: Wie kann uns, wenn wir gesund nach Hause kommen, das Hinterland für alle diese Anstrengungen, Entbehrungen und Todesängste entschädigen. Wir werden, weiß Gott wie, zurückkommen, aber wehe dem Hinterländer, der über uns lacht. [...] Die Urlauber erzählen uns von diesem Gesindel, welches den Frontsoldaten schikaniert. Ich fürchte sehr meine gewaltige Nervosität, die mit mir durchgehen wird, und ich bring so einen Höllenhund um. ${ }^{119}$

\section{Zusammenfassende Schlussbemerkung}

Der vorliegende Beitrag richtet sein Augenmerk nicht auf die oftmals untersuchten zielgerichteten Aktivitäten innerhalb militärischer Verbände, sondern versteht sich als ein Versuch in die Richtung der Sichtbarmachung und Betonung der Bedeutung von Emotionen für Soldaten an der Front. Er konzentriert sich also auf ein innerhalb der Soziologie in zweifacher Hinsicht stark vernachlässigtes Forschungsgebiet. Diese Zugangsweise wurde exemplarisch im Hinblick auf sogenannte, häufig mit dem Begriff der Kameradschaft beschriebene Wir-Gefühle sowie im Hinblick auf Feindbilder und Feindseligkeit im Krieg gewählt. Vor dem Hintergrund der Figurationssoziologie von Norbert Elias, die Organisationen wie das Militär nicht als ruhende Objekte, sondern als „Geflechte von Menschen“ versteht, wurde gezeigt, was deutschsprachige k.u.k. Soldaten im Krieg nach den Deutungen der ausgewählten Regimentsgeschichten und Ego-Dokumente in ihren jeweiligen Einheiten eigentlich aneinander bindet oder auch voneinander

117 Kuhar 2008/2009, S. 183-184.

118 Ebd., S. 185.

119 Hartinger 2012, S. 126. 
Wir-Gefühle, Feindbilder und Feindseligkeit bei deutschsprachigen k.u.k. Soldaten

trennt und welche Rolle die verschiedenen Machtbalancen (Stichwort: vertikale Loyalität) in diesem Kontext spielen. In diesem Sinne versteht sich dieser Beitrag nicht zuletzt als ein Plädoyer für das Hinzuziehen ganz unterschiedlicher Quellen mit ihren jeweiligen spezifischen Entstehungszusammenhängen und Deutungen. ${ }^{120} \mathrm{Ja}$, diese „Quellentriangulation“ erscheint vielmehr unerlässlich für eine fruchtbare emotionssoziologische Analyse zu sein:

Im Genre der Regimentsgeschichten werden die einzelnen militärischen Einheiten als durch Vertrauensbeziehungen zusammengehaltene homogene Gruppen gezeichnet. Soziale Distanz wird nicht thematisiert. Wir erfahren viel über die gültigen moralischen Werte und Codes, also wie Beziehungen im Heer sein sollten und wie sich Offiziere und Mannschaften im Krieg verhalten sollten, aber wenig über „Abweichungen“ von diesen Codes und wenig darüber, wie WirGefühle, Feindbilder und Feinseligkeit entstehen sowie gegen wen sie sich richten. Während also in offiziellen und semi-offiziellen Darstellungen „Kameradschaft“ als eine der Tugenden von Soldaten eingefordert, beschworen und mitunter verherrlicht wird, zeichnen Autobiographien und Tagebücher ein anderes, teilweise differenzierteres Bild von Kameradschaft. In den autobiographischen Manuskripten wird die Bedeutung einzelner Kameraden für den Affekthaushalt stark unterstrichen, insbesondere der Tod von Kameraden, denen man sich emotional verbunden fühlt, wirkt sich auf die Stimmungslage sehr negativ aus. Vertrauen und Bindungsgefühle wiederum zeigen sich vorrangig Kameraden gegenüber, denen man sich in regionaler Hinsicht verbunden fühlt. Gerade diese regionalen Zugehörigkeiten geben den Soldaten fern ab der Heimat Sicherheit und Geborgenheit. ${ }^{121}$ Die Komplexität von Wir-Gefühlen, die durch gemeinsam geteilte Erfahrungen gestärkt, aber auch geschwächt werden können, ${ }^{122}$ sowie die der Beziehung zwischen Offizieren und Soldaten einerseits und den einfachen Mannschaftssoldaten untereinander andererseits zeigt sich nicht in den Regi-

120 In diesem Zusammenhang spielen folgende Überlegungen eine zentrale Rolle: Wann wurde das Erlebte verschriftlicht? Wer sind die Adressaten und Adressatinnen der Schriften? Welche Form der Erinnerung ist unter den jeweiligen politischen und gesellschaftlichen Rahmenbedingungen beispielsweise in der unmittelbaren Nachkriegszeit oder zur Zeit des österreichischen Ständestaates „legitim“? Worüber darf also überhaupt berichtet werden? Und spezifischer auf meine Fragestellung bezogen: Welche Gefühle dürfen überhaupt zum Ausdruck gebracht werden (Frage der Gefühlsregeln)? 121 Ausführlich wurde dieser Aspekt in Kuzmics / Haring 2013 herausgearbeitet. Selbstredend geht es in der multiethnischen k.u.k. Armee nicht nur um regionale, sondern auch um sprachliche Zugehörigkeiten; Mehrfachloyalitäten - regionale, religiöse und sprachliche - sind hier die Norm und nicht die Abweichung.

Diese regionale Dimension betonte jüngst im Hinblick auf das Deutsche Kaiserreich Sönke Neitzel, der festhält, dass in den Briefen und Tagebüchern deutscher Soldaten die „regionale Identität immer noch spürbar“ sei (vgl. Neitzel 2016, S. 11).

122 Vgl. hier exemplarisch das Verhältnis von Obergottsberger und Bakic. 
mentsgeschichten, aber in vielen der analysierten Passagen in den Tagebüchern und autobiographischen Manuskripten.

Letztendlich gehört bisweilen auch der „Feind“, die Russen oder die Italiener, dem „Wir“ im Sinne einer „Schicksalsgemeinschaft“ als „Frontgemeinschaft“ an: Die Soldaten - Freund wie Feind - erfrieren, verhungern, verirren sich in den Winterstürmen oder sterben im feindlichen Feuer. Die für die „Gemeinschaft-“ und/oder „Freund-Kategorie“ bedeutsamen Emotionen - Vertrauen, Bindungsgefühle, Stolz und Mitleid - erstrecken sich also in der Komplexität des Frontalltags, die sich in den unterschiedlichen Quellen in zahlreichen Facetten offenbart, häufig nicht auf die Gesamtheit der in den jeweiligen Einheiten eingesetzten und miteinander interagierenden Soldaten, sondern weisen wie die gemeinhin mit Feinden assoziierten Emotionen Angst, Ärger, Wut, Zorn, Ekel, Abscheu, Verachtung und Hass zahlreiche Nuancen und Differenzierungen auf. Die Grenzen zwischen dem Wir - den k.u.k. Soldaten - und den Anderen - den sogenannten Fremden in Form der Zivilbevölkerung und den sogenannte Feinden, beispielsweise den Russen oder den Italienern, - erweisen sich als brüchig, verschwimmen bisweilen und werden in der sozialen Wirklichkeit des Frontlebens von den Akteuren immer von Neuem gezogen. Das Hinzuziehen nichtdeutschsprachiger Ego-Dokumente von k.u.k. Soldaten im Ersten Weltkrieg im Rahmen eines internationalen Teams bzw. Forschungsprojektes würde wohl weitere wichtige Differenzierungen und Nuancierungen im Hinblick auf $A f$ fekthaushalt, Gefühlregeln und moralische Codes der Soldaten herausarbeiten können. Nicht zuletzt vor dem Hintergrund der „Habsburgerarmee“ als ein mögliches historisches Laboratorium für gegenwärtige multi-kulturell und multiethnisch zusammengesetzte nationale und internationale Streitkräfte erscheint mir dies ein sehr lohnendes und dringliches Forschungsvorhaben zu sein, das nicht nur die emotionssoziologische Forschung im engeren Sinne bereichern könnte.

Letztlich kann eine emotionssoziologische Perspektive meines Erachtens zu einer Militärgeschichte von unten sowie zur Alltagsgeschichte des Krieges einen wichtigen Beitrag leisten. Sie unterstreicht darüber hinaus die Bedeutung von Emotionen als Motor für soziales Handeln und für die soziale Konstruktion der Wirklichkeit im Krieg sowie deren Stellenwert für das „Erinnern“ an den Krieg.

\section{Literaturverzeichnis}

Bauman, Zygmunt, „Moderne und Ambivalenz“, in: Bielefeld, Ulrich (Hrsg.), Das Eigene und das Fremde. Neuer Rassismus in der Alten Welt? Hamburg, Hamburger Edition, 1998, S. 23-49.

Bernsdorf, Wilhelm, „Kameradschaft“, in: Wörterbuch der Soziologie. Herausgegeben von Wilhelm Bernsdorf. Zweite, neubearbeitete und erweiterte Ausgabe, Stuttgart, Ferdinand Enke, 1969, S. 527-530. 
Wir-Gefühle, Feindbilder und Feindseligkeit bei deutschsprachigen k.u.k. Soldaten

Buschmann, Nikolaus / Murr, Karl Borromäus, „,Treue‘ als Forschungskonzept? Begriffliche und methodische Sondierungen“, in: Buschmann, Nikolaus / Murr, Karl Borromäus (Hrsg.), Treue. Politische Loyalität und militärische Gefolgschaft in der Moderne, Göttingen, Vandenhoeck \& Ruprecht, 2008, S. 11-35.

Cole, Laurence, „Militärische Loyalität in der späten Habsburgermonarchie“, in: Buschmann, Nikolaus / Murr, Karl Borromäus (Hrsg.), Treue. Politische Loyalität und militärische Gefolgschaft in der Moderne, Göttingen, Vandenhoeck \& Ruprecht, 2008, S. 347-376.

Collins, Randall, Violence. A Micro-Sociological Theory, Princeton-Oxford, Princeton University Press, 2008.

Collins, Randall, Konflikttheorie. Ausgewählte Schriften, Wiesbaden, Springer VS, 2012.

Deák, István, Der K. (u.) K. Offizier 1848-1918, Wien / Köln / Weimar, Böhlau, 1991.

Düffler, Jost, „Die geplante Erinnerung. Der Historikerboom um den Ersten Weltkrieg“, in: Osteuropa 64, 2-4, 2014, S. 351-368.

Ferguson, Niall, Krieg der Welt. Was ging schief im 20. Jahrhundert? Aus dem Englischen von Klaus-Dieter Schmidt und Klaus Binder, Berlin, Propyläen, 2006.

Friedman, Robi, „Die Gruppe der Soldaten-Matrix, in: Gruppenpsychotherapie und Gruppendynamik. Zeitschrift für Theorie und Praxis der Gruppenanalyse, 51/3, 2015a, S. 191-205.

Fröhlich, Hermann, Geschichte des steirischen k.u.k. Infanterie-Regimentes Nr. 27 für den Zeitraum des Weltkrieges 1914-1918, Band 1, Innsbruck, Wagner'sche UniversitätsBuchdruckerei, 1937.

Fröhlich, Hermann, Geschichte des steirischen k.u.k. Infanterie-Regimentes Nr. 27 für den Zeitraum des Weltkrieges 1914-1918, Band 2, Innsbruck, Wagner'sche UniversitätsBuchdruckerei, 1937a.

Grünwald, Stefan / Hauer, Nora / Huber, Martina / Stummer, Claudia, „Multinationalität, Feindbilder und deren Verbreitung“, in: Haring, Sabine A. / Kuzmics, Helmut (Hrsg.), Krieg \& Emotionen. Der Erste Weltkrieg der k.u.k. Armee in autobiographischen Dokumenten. Ergebnisse des Forschungspraktikums 2009/2010, Graz, o. V., 2009/2010, S. 223-259.

Hämmerle, Christa (Hrsg.), Des Kaisers Knechte. Erinnerungen an die Rekrutenzeit im k.(u.)k. Heer 1868 bis 1914. Herausgegeben und erläutert von Christa Hämmerle, Wien / Köln / Weimar, Böhlau Verlag, 2012.

Hämmerle, Christa, „Den Militärdienst erinnern - eine Einleitung“, in: Hämmerle, Christa (Hrsg.), Des Kaisers Knechte. Erinnerungen an die Rekrutenzeit im k.(u.)k. Heer 1868 bis 1914. Herausgegeben und erläutert von Christa Hämmerle, Wien / Köln / Weimar, Böhlau Verlag 2012a, S. 7-27.

Hämmerle, Christa, Heimat/Front. Geschlechtergeschichte/n des Ersten Weltkriegs in Österreich-Ungarn, Wien / Köln / Weimar, Böhlau, 2014.

Haring, Sabine A., „Der ,Kampf‘ sozialer Gruppen als treibende Kraft der Geschichte. Die Bedeutung und Funktion von Konflikten im Werk von Ludwig Gumplowicz“, in: Deutschmann, Peter / Munz, Volk / Pavlenko, Ol'ga (Hrsg.), Konfliktszenarien um 1900: politisch - sozial - kulturell. Die österreichisch-ungarische Monarchie und das russische Zarenreich um 1900, Wien, 2011, S. 19-41.

Haring, Sabine A. / Kuzmics, Helmut, „Einleitung“, in: Kuzmics, Helmut / Haring, Sabine A., Emotion, Habitus und Erster Weltkrieg. Soziologische Studien zum militäri- 
schen Untergang der Habsburger Monarchie im Ersten Weltkrieg, Göttingen, Vandenhoeck \& Ruprecht, 2013, S. 13-56.

Haring, Sabine A., ,Wir-Gefühle, Feindbilder und Feindseligkeit“, in: Kuzmics, Helmut / Haring, Sabine A., Emotion, Habitus und Erster Weltkrieg. Soziologische Studien zum militärischen Untergang der Habsburger Monarchie im Ersten Weltkrieg, Göttingen, Vandenhoeck \& Ruprecht, 2013, S. 269-468.

Haring, Sabine A., ,,Kameradschaft' in der Habsburgermonarchie. Eine emotionssoziologische Annäherung“, in: LiTheS. Zeitschrift für Literatur- und Theatersoziologie, hg. von Beatrix Müller-Kampel und Helmut Kuzmics, Nr. 10 (März 2014), Krieg, Mythos und Literatur, S. 54-79.

Haring, Sabine A., „Between the topos of a ,forgotten war' and the current memory boom: Remembering the First World War in Austria“, in: Ziino, Bart (Hrsg.), Remembering the First World War, London / New York, 2015, S. 207-222.

Haring, Sabine A., „Wir-Gefühle im Ersten Weltkrieg unter einer emotionssoziologischen Perspektive“, in: Gruppenpsychotherapie und Gruppendynamik. Zeitschrift für Theorie und Praxis der Gruppenanalyse, 51/3, 2015a, S. 174-190.

Hartinger, Franz Matthias, Die Kriegstagebücher des Franz Matthias Hartinger, Offizierstellvertreter im k.k. Landsturm-Infanterie-Regiment Laibach No. 27, 1915-1918. Herausgegeben von Volker Rutte, Graz, Eigenverlag, 2012.

Haslinger, Peter / Puttkamer, Joachim von, „Staatsmacht, Minderheit, Loyalität“, in: Haslinger, Peter / Puttkamer, Joachim von (Hrsg.), Staat, Loyalität und Minderheiten in Ostmittel- und Südosteuropa 1918-1941, München, Oldenbourg, 2007, S. 1-16.

Heidbrink, Horst / Lück, Helmut E. / Schmidtmann, Heide, Psychologie sozialer Beziehungen, Stuttgart, Kohlhammer, 2009.

Herberg-Rothe, Andreas, Der Krieg. Geschichte und Gegenwart, Frankfurt am Main / New York, Campus Verlag, 2003.

Hillmann, Karl-Heinz, Wörterbuch der Soziologie, 5. völlig überarbeitete und erweiterte Auflage, Stuttgart, Kröner, 2007.

Hochschild, Arlie Russell, Das gekaufte Herz. Die Kommerzialisierung der Gefühle, Frankfurt am Main / New York, Campus, 1990.

Imbusch, Peter, „Der Gewaltbegriff“, in: Heitmeyer, Wilhelm / Hagan, John (Hrsg.), Internationales Handbuch der Gewaltforschung, Wiesbaden, Westdeutscher Verlag, 2002, S. 26-57.

Jeismann, Michael, „Propaganda“, in: Hirschfeld, Gerhard / Krumeich, Gerd / Renz, Irina (Hrsg.), Enzyklopädie Erster Weltkrieg. Aktualisierte und erweiterte Studienausgabe, Paderborn / München / Wien / Zürich, Schöningh, 2009, S. 198-209.

Kühne, Thomas, Kameradschaft. Die Soldaten des nationalsozialistischen Krieges und das 20. Jahrhundert, Göttingen, Vandenhoeck \& Ruprecht, 2006.

Kuhar, Lovro (Pseud. Prežihov Voranc), Doberdò. Slowenischer Antikriegsroman (Doberdob. Vojni roman slovenskega naroda. Ljubljana 1940). Aus dem Slowenischen von Karin Almasy (Teile 2 und 4) und Klaus Detlef Olaf (Teile 1 und 3), Klagenfurt / Celovec; Ljubljana / Laibach; Wien / Dunaj, Hermagoras / Mohorjeva založba, 2008 / 2009.

Kuzmics, Helmut / Haring, Sabine A., Emotion, Habitus und Erster Weltkrieg. Soziologische Studien zum militärischen Untergang der Habsburger Monarchie im Ersten Weltkrieg, Göttingen, Vandenhoeck \& Ruprecht, 2013. 
Kuzmics, Helmut, „Entwicklungslinien und Typen des habsburgischen Offiziershabitus vor 1914 im Spiegelbild schöner Literatur", in: Kuzmics, Helmut / Haring, Sabine A., Emotion, Habitus und Erster Weltkrieg. Soziologische Studien zum militärischen Untergang der Habsburger Monarchie im Ersten Weltkrieg, Göttingen, Vandenhoeck \& Ruprecht, 2013a, S.137-167.

Kuzmics, Helmut, „Der k.u.k. Armeehabitus im Ersten Weltkrieg“, in: Kuzmics, Helmut / Haring, Sabine A., Emotion, Habitus und Erster Weltkrieg. Soziologische Studien zum militärischen Untergang der Habsburger Monarchie im Ersten Weltkrieg, Göttingen, Vandenhoeck \& Ruprecht, 2013b, S. 169-268.

Leidinger, Hannes, „Eskalation der Gewalt“, in: Leidinger, Hannes / Moritz, Verena / Moser, Karin / Dornik, Wolfram (Hrsg.), Habsburgs schmutziger Krieg. Ermittlungen zur österreichisch-ungarischen Kriegsführung 1914-1918, St. Pölten / Salzburg / Wien, Residenz Verlag, 2014, S. 51-91.

Lein, Richard, Pflichterfüllung oder Hochverrat? Die tschechischen Soldaten ÖsterreichUngarns im Ersten Weltkrieg (= Europa Orientalis, herausgegeben vom Institut für Osteuropäische Geschichte an der Universität Wien, Band 9), Wien, Lit, 2011.

Lichem, Heinz von, Spielhahnstoß und Edelweiß. Die Friedens- und Kriegsgeschichte der Tiroler Hochgebirgstruppe „Die Kaiserschützen“ von ihren Anfängen bis 1918: k.k. Tiroler Landesschützen-Kaiserschützen-Regimenter Nr. I - Nr. II - Nr. III, Graz I Stuttgart, Leopold Stocker Verlag, 1977.

Liedtke, Max, „Zur Entstehung von Feind- und Freundbildern am Beispiel des deutschsprachigen Schulbuchs. Historische Darstellung und evolutionstheoretische Hintergründe“", in: Djurović, Arsen / Matthes, Eva (Hrsg.), Freund- und Feindbilder in Schulbüchern. Concepts of Friends and Enemies in Schoolbooks, Bad Heilbrunn, Julius Klinkhardt, 2010, S. 19-32.

Lukan, Walter / Peyfuss, Max D., „Jeder Schuß ein Russ', jeder Stoß ein Franzos. Kriegspropaganda auf Postkarten“, in: Weigel, Hans / Lukan, Walter / Peyfuss, Max D., Jeder Schuss ein Russ', jeder Stoß ein Franzos. Literarische und graphische Kriegspropaganda in Deutschland und Österreich 1914-1918, Wien, Edition Christian Brandstätter 1983, S. 32-47.

Meteling, Wencke, Ehre, Einheit, Ordnung. Preußische und französische Städte und ihre Regimenter im Krieg, 1870/71 und 1914-19, Baden-Baden, Nomos, 2010.

Neitzel, Sönke, „Der Erste Weltkrieg - Regionale Perspektiven im europäischen Kontext. Einführende Bemerkungen“, in: Elvert, Jürgen et al. (Hrsg.), Historische Mitteilungen 28, Schwerpunkt: Der Erste Weltkrieg - Regionale Perspektiven, Stuttgart, Franz Steiner Verlag, 2016, S. 7-14.

Obergottsberger, Franz, Ich hörte noch die Nachtigal. Die Lebensgeschichte des Franz. Obergottsberger 1895-1979. Band II: Krieg und Gefangenschaft, bearbeitet und als Manuskript veröffentlicht von seinem Sohn Hugo Obergottsberger. Dokumentation lebensgeschichtlicher Aufzeichnungen, Institut für Wirtschafts- und Sozialgeschichte, Universität Wien, o. J.

Pomberger, Gottlieb, Wiedergabe der Erlebnisse und Begebenheiten im Weltkriege vom Jahre 1914 bis 1918 des gefertigten Gottlieb Pomberger aus Gosau Nr. 92, Dokumentation lebensgeschichtlicher Aufzeichnungen, Institut für Wirtschafts- und Sozialgeschichte, Universität Wien, o. J. 
Reiß, Siegfried (Hrsg.), Ehrenbuch der Heßer: Geschichte des altösterreichischen Infanterie-Regimentes Freiherr von Heß Br. 49, 1715-1918, Band 2: Der Weltkrieg 19141918, Wien, Schöler, 1926.

Remarque, Erich Maria, Im Westen nichts Neues. Roman. Mit Materialien und einem Nachwort von Tilman Westphalen, 19. Auflage, Köln, Kiepenheuer \& Witsch, 1996.

Rothenberg, Gunther E., „The Habsburg Army in the First World War: 1914-1918“, in: The Habsburg Empire in World War I. Essays on the Intellectual, Military, Political and Economic Aspects of the Habsburg War Effort. Herausgegeben von Robert A. Kann, Bela K. Kiraly und Paula S. Fichtner, New York, Columbia University Press, 1977, S. 73-86.

Royer, Matthias, Schilderungen meiner Erlebnisse als Soldat und Kriegsgefangener im ersten Weltkrieg in Russland vom 27.02.1914 bis 19.10.1920, o. J.

Scheer, Tamara, „K.u.k. Regimentssprachen: Institutionalisierung der Sprachenvielfalt in der Habsburgermonarchie in den Jahren 1867/8-1914“, in: Ehlers, Klaas-Hinrich / Nekula, Marek / Niedhammer, Martina / Scheuringer, Hermann (Hrsg.), Sprache, Gesellschaft und Nation in Ostmitteleuropa. Institutionalisierung und Alltagspraxis (=Bad Wiesseer Tagungen des Collegium Carolinum, Band 35), Göttingen, Vandenhoeck \& Ruprecht, 2014, S. 75-92.

Scheff, Thomas J., Bloody Revenge. Emotions, Nationalism, and War, Boulder, Colorado, Westview Press, 1994.

Scherke, Katharina, Emotionen als Forschungsgegenstand der deutschsprachigen Soziologie, Wiesbaden, VS Verlag für Sozialwissenschaften, 2009.

Schmölzer, Michael / Zaunbauer, Wolfgang, „Das große Gedenken“, in: Wiener Zeitung vom Sa./So., 4./5. Jänner 2014, S. 10-11.

Senge, Konstanze, „Die Wiederentdeckung der Gefühle. Zur Einleitung“, in: Senge, Konstanze / Schützeichel, Rainer, Hauptwerke der Emotionssoziologie, Wiesbaden, Springer, 2013, S. 11-37.

Sorg, Werner, Das Wesen der Kameradschaft. Unveröffentlichte Diplomarbeit. Theresianische Militärakademie. Wiener Neustadt, 2004.

Stegmann, Natali, Kriegsdeutungen - Staatsgründungen - Sozialpolitik. Der Helden- und Opferdiskurs in der Tschechoslowakei 1918-1948, München, Oldenbourg, 2010.

Stietencron, Heinrich von, „Töten im Krieg. Grundlagen und Entwicklungen“, in: Stietencron, Heinrich von / Rüpke, Jörg (Hrsg.), Töten im Krieg, Freibur im Breisgau / München, Verlag Karl Alber, 1995, S. 17-56.

Stouffer, Samuel A. et al., The American Soldier. Volume 2. Combat and Its Aftermath, Princeton, New York, Princeton University Press, 1949.

Tangney, June Price / Stuewig, Jeff / Mashek, Debra J. „Moral Emotions and Moral Behavior“, in: Annual Review of Psychology, 58, 2007, S. 345-372.

Turner, Jonathan H. / Stets, Jan E., „Moral Emotions“, in: Stets, Jan E. / Turner, Jonathan H. (Hrsg.), Handbook of the Sociology of Emotions, New York, Springer Science \& Business Media, 2007, S. 544-566.

Vogelsang, Ludwig Freiherr von, Das steirische Infanterieregiment Nr. 47 im Weltkrieg. Zum 250. Errichtungsjahr des Regiments, Band 1 and 2, Graz, Leykam, 1932 und 1932a. 
Waldstätten-Zipperer, Josef / Seifert, Josef, Die Deutschmeister. Taten und Schicksale der Infanterieregiments Hoch- und Deutschmeister Nr. 4 insbesondere im Weltkriege, Wien, Österreichische Staatsdruckerei, 1928.

Wagener, Sybil, Feindbilder. Wie kollektiver Hass entsteht, Berlin, Quadriga-Verlag, 1999.

Wassmann, Claudia, Die Macht der Emotionen. Wie Gefühle unser Denken und Handeln beeinflussen, Darmstadt, Wissenschaftliche Buchgesellschaft, 2002.

Wyrwa, Ulrich, „Zum Hundertsten nichts Neues. Deutschsprachige Neuerscheinungen zum Ersten Weltkrieg (Teil II)“, in: Zeitschrift für Geschichtswissenschaft, 64, 7-8, 2016, S. 683-702.

\section{Internetquellen}

Uhl, Heidemarie, „,Der Erste Weltkrieg im Gedächtnis Österreichs und (Zentral-)Europas - Gedächtnistraditionen in (trans)nationaler Perspektive“, online abrufbar unter: http://www.bmeia.gv.at/fileadmin/user_upload/bmeia/media/3-Kulturpoliti sche_Sektion_-_pdf/Themen_Dateien/Grundlagenpapier_1914_-_2014.pdf, 2013, S. 3739.

Sabine A. Haring, Department of Sociology, University of Graz, Universitätsstraße 15/ G4, 8010 Graz, Austria, e-mail: sabine.haring@uni-graz.at

Citation: Haring, Sabine A.: „Wir-Gefühle, Feindbilder und Feindseligkeit bei deutschsprachigen k.u.k. Soldaten im Ersten Weltkrieg. Eine emotionssoziologische Perspektive“, in: Kallhoff, Angela / Schulte-Umberg, Thomas (eds.): Moralities of Warfare and Religion (J-RaT 2018 / 1) pp. 53-87.

Datum der Publikation: 16.07.2018 